Chapter 9

\title{
Nanostructured \\ Detector Technology for \\ Optical Sensing Applications
}

\author{
Ashok K. Sood, Nibir K. Dhar, Dennis L. Polla, \\ Madan Dubey and Priyalal Wijewarnasuriya \\ Additional information is available at the end of the chapter \\ http://dx.doi.org/10.5772/58349
}

\section{Introduction}

This Chapter covers recent advances in nanostructured based detector technology, materials and devices for optical sensing applications. The authors have many years of experience working nanotechnologies that include a variety of semiconductors and other advanced materials such as $\mathrm{GaN}, \mathrm{ZnO}, \mathrm{Si} / \mathrm{SiGe}, \mathrm{CNT}$ and Graphene for optical sensing applications.

Optical sensing technology is critical for defense and commercial applications including optical communication. Advances in optoelectronics materials in the UV, Visible and Infrared, using nanostructures, and use of novel materials such as CNT and Graphene have opened doors for new approaches to apply device design methodology that are expected to offer enhanced performance and low cost optical sensors in a wide range of applications.

We will cover the UV band (200-400 nm) and address some of the recent advances in nanostructures growth and characterization using $\mathrm{GaN} / \mathrm{AlGaN}, \mathrm{ZnO} / \mathrm{MgZnO}$ based technologies and their applications. We will also discuss nanostructure based Si/SiGe technologies (400-1700 $\mathrm{nm}$ ) that will cover various bands of interest in visible-near infrared for detection and optical communication applications. The chapter will also discuss some of the theoretical and experimental results in these detector technologies.

Recent advancements in design and development of CNT based detection technologies have shown promise for optical sensor applications. We will present theoretical and experimental results on these device and their potential applications in various bands of interest. 


\section{UV, Visible and infrared spectrum and bands of interest}

The Ultraviolet spectrum has been of interest for a variety of sensors for defense and commercial applications. The UV band is from 250-400 nanometers as shown in the figure 1 . This band can be further divided into UVA and UVB bands. Each of these bands has applications for sensors, detectors and LED applications.

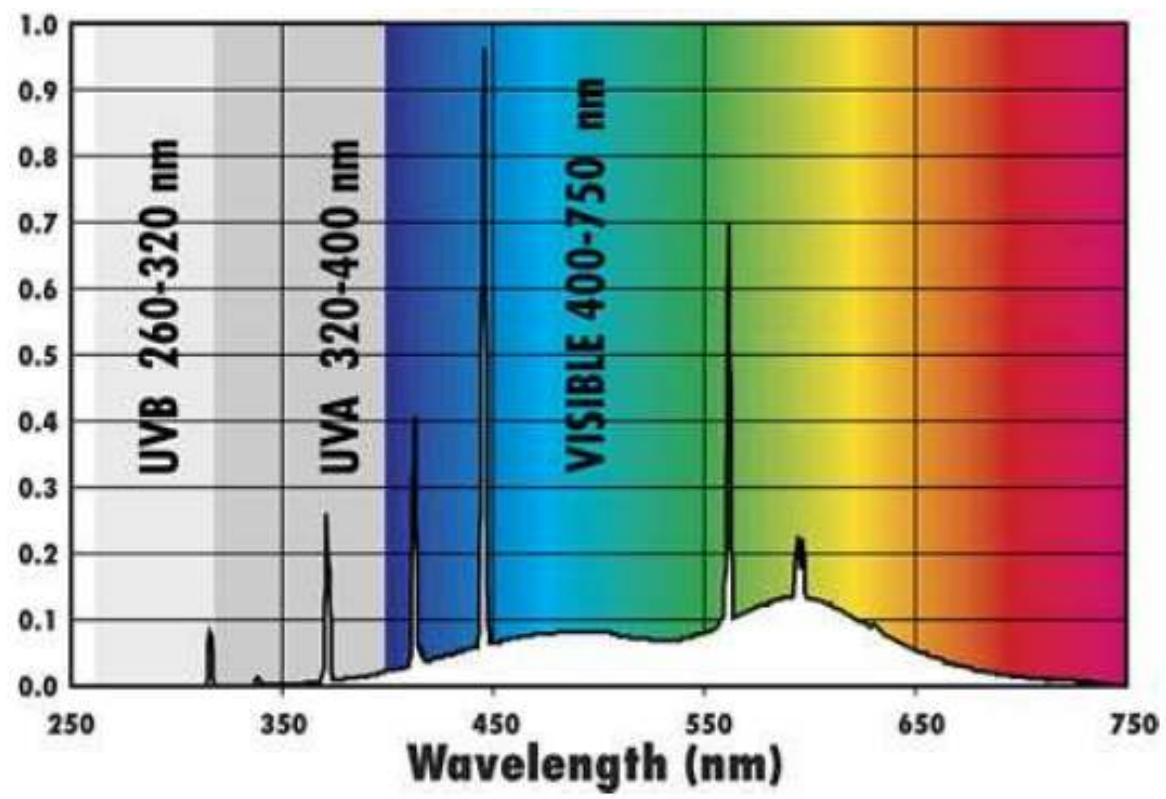

Figure 1. Overview of UV and Visible Spectral Band [1]

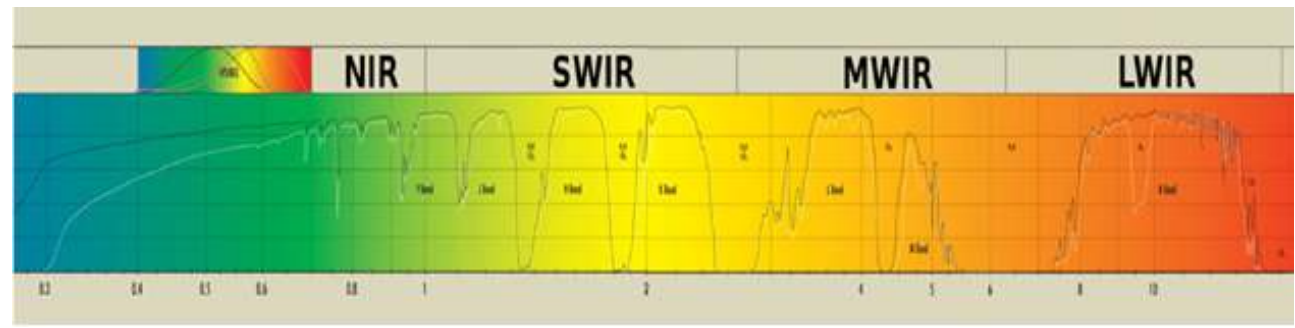

Figure 2. Definition of IR Spectral Band [1]. 
The word "infrared" refers to a broad portion of the electromagnetic spectrum that spans a wavelength range from $1.0 \mathrm{um}$ to beyond 30 um everything between visible light and microwave radiation. Much of the infrared spectrum is not useful for ground- or sea-based imaging because the radiation is blocked by the atmosphere. The remaining portions of the spectrum are often called "atmospheric transmission windows," and define the infrared bands that are usable on Earth. The infrared spectrum is loosely segmented into near infrared (NIR, 0.8-1.1um), short wave infrared (SWIR, 0.9-2.5um), mid wave infrared (MWIR, 3-5um), long wave infrared (LWIR, 8-14um), very long wave infrared (VLWIR, 12- 25um) and far infrared (FIR, > 25um), as shown in Figure 2. The MWIR- LWIR wavebands are important for the imaging of objects that emit thermal radiation, while the NIR-SWIR bands are good for imaging scenes that reflect light, similar to visible light.

Since NIR and SWIR are so near to the visible bands, their behavior is similar to the more familiar visible light. Energy in these bands must be reflected from the scene in order to produce good imagery, which means that there must be some external illumination source. Both NIR and SWIR imaging systems can take advantage of sunlight, moonlight, starlight, and an atmospheric phenomenon called "nightglow," but typically require some type of artificial illumination at night. In lieu of photon starved scenes, arrays of infrared Light Emitting Diodes (LEDs) can provide a very cost effective solution for short-range illumination. However, achieving good performance at distances of over hundreds of meters requires more directed illumination, such as a focused beam from a laser or specialized spotlight, although special consideration of eye-safety issues is required.

\section{Ultraviolet nanostructured detector array development}

\subsection{Applications of UV imaging technology}

Imagery for identification of targets at various distances uses visible cameras, image intensifiers, shortwave IR cameras and long wave uncooled cameras. Each have distinct advantages and disadvantages and are each useful under specific sets of conditions such as light level, thermal conditions, and level of atmospheric obscuration. The shortest wavelength is desired for spatial resolution which allows for small pixels and large formats. [2- 6]

Visible cameras, if adequate light level is present, can provide high resolution, but for long range identification even under moonlit and starlit illuminations, long integration times and large optics are required and dust, smoke and fog easily defeat a single visible camera. Image intensifiers and SWIR cameras are useful in many conditions as the SWIR penetrates fog easily but requires fairly clear night skies for the upper atmospheric airglow light source, and image intensifiers require a certain level of celestial (starlight, moonlight) or light pollution irradiance. Both the SWIR and image intensifiers are limited by the diffraction resolution of the NIR to SWIR wavelengths [5-6]. 
For optimal resolution, the visible or ultraviolet spectrum is preferable; however, active (laser) illumination is required for long-range night imaging. Covert UV illumination is preferred over the visible and the atmosphere transmits fairly well at the longer UV wavelengths. The covert active system for high-resolution identification modeled in this paper consists of a UV laser source and a silicon CCD, AlGaN or AlGaN APD focal plane array with pixels as small as 4 microns that are spectrally tuned for the solar-blind region of the UV spectrum. The solarblind region is optimal as virtually all of the solar radiation is absorbed at the higher altitudes leaving a pitch dark terrain even in bright day, yet for sea-level path lengths of $1 \mathrm{~km}$ and shorter; the UV atmospheric transmittance is still acceptable.

This combination is ideal for exploitation by a UV illuminator and UV FPA sensor. Current UV lasers can provide either continuous or pulsed energy at levels detectable by solarblind UV detectors under relatively small optics and at $30 \mathrm{~Hz}$ frame rates, providing realtime high-resolution (on the order of $1 \mathrm{~cm}$ at $1 \mathrm{~km}$ ) imagery. At these illumination levels and target ranges, both standard PN, PIN and APD UV detectors and silicon CCD's can be used for target identification. The model has been developed and used to include the combined effects of detector and electronics, atmospheric transmittance and UV background radiance, target size, range and reflectance, and UV laser attributes to simulate and predict both $\mathrm{CW}$ and pulsed laser imaging performance and to assist in the design of this prototype system [6].

\subsubsection{Model development for passive and active UV systems design}

The general equations for SNR prediction for laser illumination and APD are derived.

$$
S N R=\frac{G e_{\text {Lret }}}{\left[F^{2} G^{2}\left(e_{\text {Lret }}+e_{b k}+e_{d k}\right)+\left(\tilde{e}_{n, a m p}\right)^{2}\right]^{1 / 2}}
$$

Where G is the APD gain, $\mathrm{F}$ is the excess noise, the noise electron terms are the laser return shot noise, the scene noise, the dark current noise and the amp noise

Two special and frequently occurring cases are (2) for the laser power noise limited case and (3) for the amp noise limited case:

$$
\begin{aligned}
& S N R=\frac{\left[e_{\text {Lret }}\right]^{1 / 2}}{F} \\
& S N R=\frac{G e_{\text {Lret }}}{\left(\tilde{e}_{n, \text { amp }}\right)}
\end{aligned}
$$

The laser return in electrons for $\mathrm{cw}$ assuming lambertian reflection is: 


$$
e_{\text {Lret }, t i}=\left[t_{i} \eta \tau_{o}\right]\left[P_{L c w} \frac{\lambda}{h c}\right] \tau_{a} \frac{\Omega_{\text {pix }}}{\Omega_{L}} \rho_{\text {tar }} \tau_{a}^{2} \frac{A_{o}}{\pi R^{2}}=\left[t_{i} \eta \tau_{o}\right]\left[P_{L c w} \frac{\lambda}{h c}\right] \tau_{a} \frac{4 A_{\text {pix }}}{\pi \theta_{\text {fdiv }}^{2} f^{2}} \rho_{\text {tar }} \tau_{a}^{2} \frac{A_{o}}{\pi R^{2}}
$$

Or when separated into detector/optics, atmosphere, laser and target attributes:

$$
e_{\text {Lret }, t i}=\left[t_{i} \eta \tau_{o} \frac{A_{\text {pix }} A_{o}}{\pi f^{2}}\right] \tau_{a}^{2} \frac{\left[\Phi_{\text {Lcw }}\right]}{\theta_{\text {fdiv }}^{2}} \frac{4 \rho_{\text {tar }}}{\pi R^{2}}
$$

If we allow for frame summing:

$$
e_{\text {Lret }, t i+}=N_{f s}\left[t_{i} \eta \tau_{o} \frac{A_{\text {pix }} A_{o}}{\pi f^{2}}\right] \tau_{a}^{2} \frac{\left[\Phi_{L c w}\right]}{\theta_{\text {fdiv }}^{2}} \frac{4 \rho_{\text {tar }}}{\pi R^{2}}
$$

For pulse laser operation and using $t_{\text {bin }}$ which equals $t_{\text {pulse }}$ and the number of bins per frame $\mathrm{N}_{\text {bins/f: }}$ :

$$
e_{\text {Limage }}=\left[N_{\text {bins } / f} \eta \tau_{o} \frac{A_{\text {pix }} A_{o}}{\pi f^{2}}\right] \tau_{a}^{2} \frac{P_{\text {Lpulse }}(\lambda / h c)}{\theta_{\text {fdiv }}^{2}} \frac{4 \rho_{\text {tar }}}{\pi R^{2}}
$$

\subsubsection{Systems performance metrics for UV systems design}

To model the sensor and system performance, we have assumed the pixel size for a high sensitivity, detector size of $5-20$ microns for the UV detector array. The fill factor of $70 \%$ is assumed typical for these small pixels. Typical quantum efficiencies have been assumed to be in the 70\% range for the PIN diode and APD [5-6]. The model uses as default, an amp noise of 15 electrons per frame time, a dark current of $1 \mathrm{e}-15$ amps for a 5 micron pixel or $4 \mathrm{nA} / \mathrm{cm}^{2}$ or 200 electrons or about 14 noise electrons, and scene noise is effectively zero in the solar-blind region.

The model from the MODTRAN runs shown in figure 3, the daytime irradiance in the UV is insignificant in the solar-blind region. The drop-off from 0.30 microns to 0.26 microns illustrates the requirement for a UV detector with spectral response is in the solar-blind region. Figure 4 shows the UV spectral radiance at midday and the comparative laser illumination of the target at $1 \mathrm{~km}$ for a 6 milliradian beam divergence for powers of $1 \mathrm{~mW}$ and $10 \mathrm{~mW}$. The left plot in the figure shows that the transmittance improves with longer UV wavelengths for all three levels of aerosols and is sufficient for $1 \mathrm{~km}$ lengths in our solar-blind region.

To achieve high-resolution day-night imaging and identification of targets, the following conditions and requirements must be met. While linear detection (no APD and no laser illumination) is fine for muzzle flashes and images of nearby combatants illuminated by live 
fire (a millisecond event), laser illumination is required for cold targets (facial recognition, profile recognition). A continuous laser and $33 \mathrm{msec}$ integrations are adequate if enough laser power is available. If not, a pulsed laser with nanosecond integrations and APD detectors are required to reduce atmosphere scatter and improve detector sensitivity.

\begin{tabular}{|c|c|c|c|c|c|c|c|c|c|}
\hline \multicolumn{2}{|c|}{ SPECTRAL BANDS } & \multicolumn{3}{|l|}{ LASER } & & \multicolumn{3}{|c|}{ SCENE / TARGET TIMING } & \\
\hline lam hi & $0.267 \mathrm{um}$ & lambda & \multicolumn{2}{|c|}{$0.266 \mathrm{um}$} & & $t$ transit & \multicolumn{2}{|c|}{$3.33 \mathrm{E}-06 \mathrm{sec}$} & lin overfill \\
\hline lam lo & $0.265 \mathrm{um}$ & P laser cont & \multicolumn{2}{|c|}{$0.10 \mathrm{w}$} & & & \multicolumn{2}{|c|}{$\begin{array}{l}3333 \text { nsec } \\
3.33 \text { usec }\end{array}$} & 1.25 \\
\hline lam mid & $0.266 \mathrm{um}$ & Pd laser cont & \multirow{2}{*}{\multicolumn{2}{|c|}{$\begin{array}{c}3.18 \mathrm{E}-06 \mathrm{~W} / \mathrm{cm} 2 \\
1.34 \mathrm{E}+17 \mathrm{pho} / \mathrm{sec}\end{array}$}} & & & \multicolumn{2}{|c|}{3.33 usec } & FPA FOV (ft) \\
\hline & & $\mathrm{Ph}$ laser cont & & & & N pulses/fr & \multicolumn{2}{|c|}{9999 max poss } & 5.25 \\
\hline \multicolumn{2}{|c|}{ DETECTOR / FPA } & $\mathrm{Ph}$ laser cont frame & \multicolumn{2}{|c|}{$4.46 \mathrm{E}+15$ pho/frame } & & & & & \\
\hline format & 256 & E laser pulse & \multicolumn{2}{|c|}{$5.00 \mathrm{E}-06$ joule/pulse } & & & & & \\
\hline \multirow[t]{2}{*}{ dpix } & $5 \mathrm{um}$ & Ph laser pulse & \multirow{2}{*}{\multicolumn{2}{|c|}{$\begin{array}{l}6.69 \mathrm{E}+12 \text { pho/pulse } \\
6.69 \mathrm{E}+12 \text { pho/bin }\end{array}$}} & & & & & \\
\hline & $2.50 \mathrm{E}-07 \mathrm{~cm} 2$ & $\mathrm{Ph}$ laser bin & & & & & & & \\
\hline ti cont & $33.33 \mathrm{msec}$ & div full & \multicolumn{2}{|c|}{$4 \mathrm{mrad}$} & & & & & \\
\hline$t$ bin & 20 nsec & t pulse & \multicolumn{2}{|c|}{20 nsec } & & & & & \\
\hline$t$ quench & $3000 \mathrm{nsec}$ & w pulse & \multicolumn{2}{|c|}{6 meters } & & & & & \\
\hline gain & 1 & bin and pulse rates & det based & mission based & $\operatorname{det} / \mathrm{mssion} \min$ & $\min$ in $\mathrm{kHz}$ & used $(\mathrm{Hz})$ & used $(\mathrm{kHz})$ & \\
\hline gain apd & 1 & sample rate $(\max ) \mathrm{Hz}$ & $3.31 \mathrm{E}+05$ & $3.00 \mathrm{E}+05$ & $3.00 \mathrm{E}+05$ & 300.0 & & & \\
\hline Fm noise & 1 & N pulses/frame & 11036 & 9999 & 9999 & 300.0 & 666.6 & 0.6666 & \\
\hline amp noise & $5.80 \mathrm{E}+10$ & $\mathrm{~N}$ bins/frame & 11036 & 9999 & 9999 & 500.0 & 666.6 & 0.6666 & \\
\hline tau opt & 0.90 & \multirow{2}{*}{\multicolumn{3}{|c|}{ Dlaser tar $\left(\mathrm{cm}, \mathrm{cm}^{2}\right)$}} & & & & & \\
\hline qe & 0.70 & & & & & & & & \\
\hline eta inj & 1.00 & \multicolumn{7}{|c|}{ ELECTRONS AND NOISE from laser, scene, dark current and amp } & \\
\hline fill factor & 0.75 & & electrons & electrons & electrons & noise e & noise e & noise $e$ & \\
\hline I dark & $5.00 \mathrm{E}-16$ a & & frame & for bins & per & frame & for bins & per & \\
\hline J dark & $2.00 \mathrm{E}-09 \mathrm{a} / \mathrm{cm}^{2}$ & & full int & in frame & bin & full int & in frame & bin & \\
\hline I surface & $1.00 \mathrm{E}-21 \mathrm{a}$ & e laser ret (s) & 9713.1 & 647.5 & $9.71 \mathrm{E}-01$ & 98.55 & 25.45 & $9.86 \mathrm{E}-01$ & \\
\hline Rload & $1.00 \mathrm{E}+06 \mathrm{ohm}$ & e laser ret cont & 647.5 & na & na & 25.45 & na & na & \\
\hline resp frame & $1.57 \mathrm{E}-02$ & elect scene (b) & 0 & $9.73 \mathrm{E}-17$ & $1.46 \mathrm{E}-19$ & 0.00 & 0.00 & $3.82 \mathrm{E}-10$ & \\
\hline \multirow{2}{*}{\multicolumn{2}{|c|}{$9.45 \mathrm{E}-09$}} & e dark (d) & 104 & $4.16 \mathrm{E}-02$ & $6.24 \mathrm{E}-05$ & 10.20 & 0.20 & $7.90 \mathrm{E}-03$ & \\
\hline & & e surface & $2.08 \mathrm{E}-04$ & $8.32 \mathrm{E}-08$ & $1.25 \mathrm{E}-10$ & 0.01 & 0.00 & $1.12 \mathrm{E}-05$ & \\
\hline \multicolumn{2}{|l|}{ OPTICS } & e kT amp & & & & 10.00 & 0.77 & $7.74 \mathrm{E}-03$ & \\
\hline dopt & $20 \mathrm{~cm}$ & & & & & & & & \\
\hline Aopt & 314.16 cm2 & MODE & signal & noise $s+b+d$ & noise $b+d$ & SNR $s+b+d$ & SNR $b+d$ & SNR $s+b+d$ & SNR $b+d$ \\
\hline focal length & $40 \mathrm{~cm}$ & DDLM cont & 647.5 & 29.2 & 14.3 & 22.2 & 45.3 & 44.4 & 90.7 \\
\hline fnum & 2.00 & DDLM bins sum & 647.5 & 25.4 & 0.3 & 25.4 & 2267.2 & 50.9 & 4534.3 \\
\hline
\end{tabular}

Figure 3. UV Sensor Model for evaluating UV Sensor Performance [6]

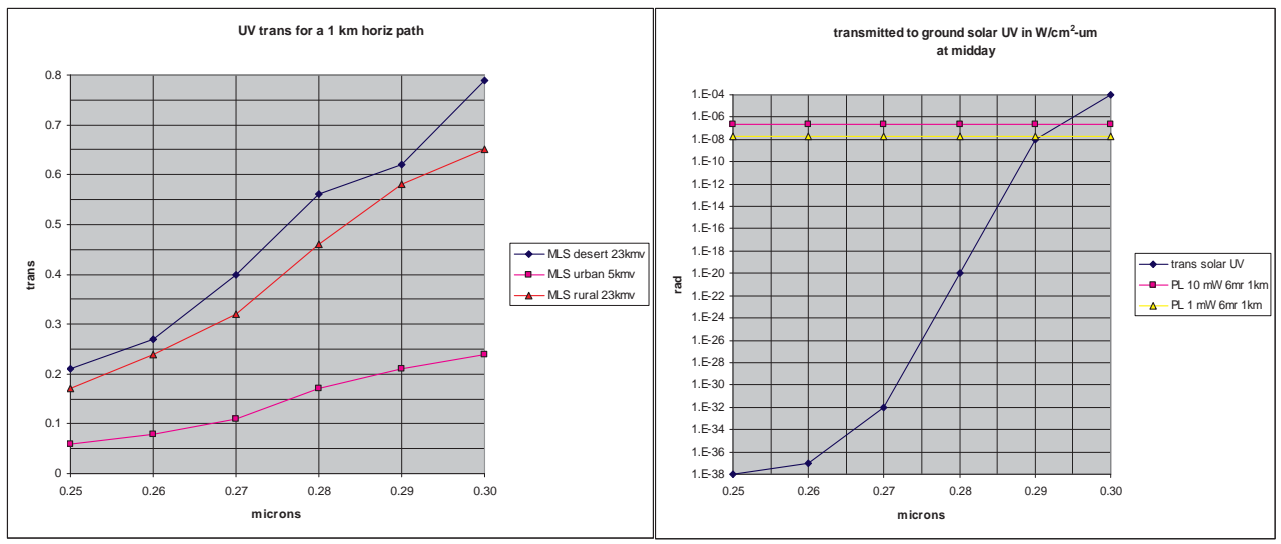

Figure 4. UV transmittance vs. wavelength for three aerosol levels (left) and UV radiance at sea level during midday and laser irradiance on the target at $1 \mathrm{~km}$ (6 mradian beam) from a $1 \mathrm{~mW}$ and $10 \mathrm{~mW}$ UV laser (right) [6] 


\section{2. $\mathrm{ZnO} / \mathrm{MgZnO}$ nanostructures for UV applications}

Zinc oxide $(\mathrm{ZnO})$ is a unique wide bandgap biocompatible material system exhibiting both semiconducting and piezoelectric properties that has a diverse group of growth morphologies. Bulk $\mathrm{ZnO}$ has a bandgap of $3.37 \mathrm{eV}$ that corresponds to emissions in the ultraviolet (UV) spectral band [7]. Highly ordered vertical arrays of $\mathrm{ZnO}$ nanowires (NWs) have been grown on substrates including silicon, $\mathrm{SiO}_{2}, \mathrm{GaN}$, and sapphire using a metal organic chemical vapor deposition (MOCVD) growth process [7]. The structural and optical properties of the grown vertically aligned $\mathrm{ZnO} \mathrm{NW}$ arrays have been characterized by scanning electron microscopy (SEM), X-ray diffraction (XRD), and photoluminescence (PL) measurements [7-10]. Compared to conventional UV sensors, detectors based on ZnO NWs offer high UV sensitivity and low visible sensitivity, and are expected to exhibit low noise, high quantum efficiency, extended lifetimes, and have low power requirements [11-12]. The Photoresponse switching properties of NW array based sensing devices have been measured with intermittent exposure to UV radiation, where the devices were found to switch between low and high conductivity states at time intervals on the order of a few seconds. Envisioned applications for such sensors/FPAs potentially include defense and commercial applications [13].

Zinc oxide is a versatile functional material that provides a biocompatible material system with a unique wide direct energy band gap and exhibits both semiconducting and piezoelectric properties. $\mathrm{ZnO}$ is transparent to visible light and can be made highly conductive by doping. Bulk $\mathrm{ZnO}$ has a bandgap of $3.37 \mathrm{eV}$ that includes emissions in the solar blind ultraviolet (UV) spectral band ( 240-280 nm), making it suitable for UV detector applications [7]. Over this wavelength range, solar radiation is completely absorbed by the ozone layer of the earth's atmosphere, so the background solar radiation at the earth's surface is essentially zero. This enhances the capability of UV sensors in missile warning systems to detect targets such as missile plumes and flames emitting in this region.

$\mathrm{ZnO}$ is the basis for the one of the richest families of nanostructures among all materials taking into accounts both structure and properties. $\mathrm{ZnO}$ growth morphologies have been demonstrated for nanowires, nanobelts, nanocages, nanocombs, nanosprings, nanorings, and nanohelixes [7]. The development of $\mathrm{ZnO}$ nanowire (NW) based UV detectors offers high UV sensitivity and low visible sensitivity for missile warning related applications. Demonstration of devices using single $\mathrm{ZnO} \mathrm{NW}$ strands has been widely reported in literature [7-16]. However, the development of reliable 2D arrays of aligned ZnO NWs has proven more challenging. The demonstration of reliable $2 \mathrm{D}$ arrays requires (1) correlation of growth process and growth parameters with the material quality of $\mathrm{ZnO} N W s,(2)$ correlation of the electrical and optical performance with growth parameters and fabrication processes, and (3) addressing system design challenges [17-18].

With conventional NW growth methods including electrochemical deposition, hydrothermal synthesis, and molecular beam epitaxy (MBE), it is generally difficult to scale up and control NW growth. Electrochemical deposition is well suited for large scale production but does not allow control over the NW orientation. Hydrothermal synthesis is a low temperature and lowcost process that allows growth of NWs on flexible substrates without metal catalysts, but the direction and morphology of the NWs cannot be well-controlled with this method [8-10]. The 
MBE method allows monitoring of the structural quality during NW growth; however, this type of synthesis often requires use of metal catalysts as a seed layer [10], which introduces undesired defects to the structure, decreasing the crystal quality [12-16]. Chemical vapor deposition (CVD) also requires catalysts at the NW tips, and using this method the tips of the grown NWs were observed to be flat, with vertical alignment.

\subsection{Characterization of $\mathrm{ZnO}$ NWs arrays grown on the various substrates}

The samples were characterized by scanning electron microscopy (SEM) utilizing a Quanta FEG 250 system, and X-ray diffraction (XRD) using Bruker D-8 Advance X-ray diffractometer with a wavelength of $1.5406 \AA$ corresponding to the $\mathrm{Cu} K \alpha$ line. In addition, photoluminescence (PL) measurements were performed at room temperature using a Linconix HeCd UV laser emitting at a wavelength of $325 \mathrm{~nm}$. A Si detector in conjunction with at lock-in amplifier and chopper were used to measure the PL from the beam reflected off the sample at the output over the desired wavelength range [18-20].
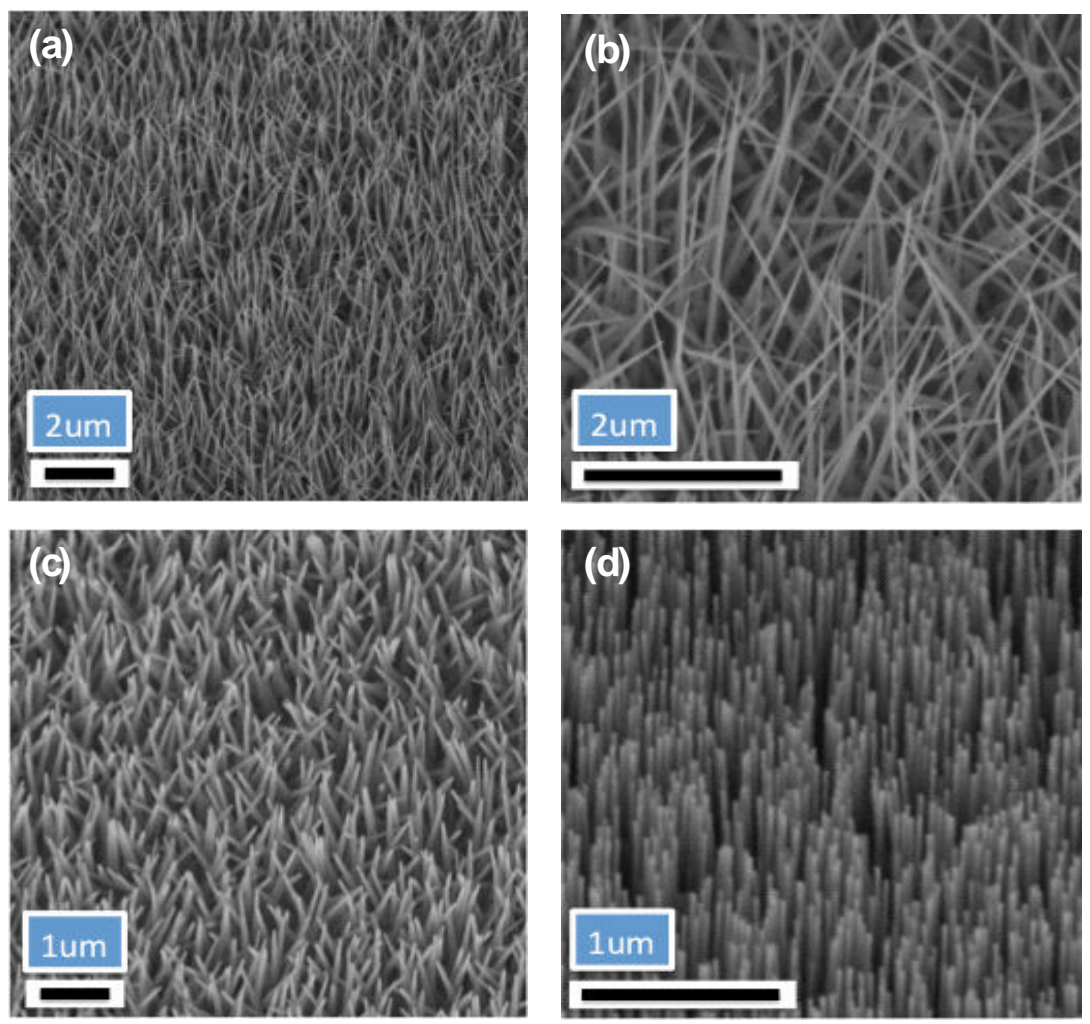

Figure 5. Scanning electron microscope (SEM) images of NWs grown on the various substrates taken at room temperature, showing NWs grown on (a) ZnO/sapphire; (b) $\mathrm{ZnO} / \mathrm{SiO}_{2} / \mathrm{p}-\mathrm{Si}$; (c) ZnO/p-Si; and (d) ZnO/GaN/sapphire.[ 20 ] 
SEM was performed to explore the NWs morphology. Figure 5 show the synthesized ZnO NWs on the various substrates, which can be generally seen to have uniform distribution density. The ZnO NWs grown on sapphire [Figure 5(a)] had approximate diameters of 50-70 $\mathrm{nm}$ and lengths in the range of 1-2 $\mu \mathrm{m}$. NWs grown on $\mathrm{SiO}_{2}$ [Figure 5(b)] had diameters of 150-200 nm and lengths of 1-2 $\mu \mathrm{m}$, and were the least vertically oriented and associated with a relatively high lattice mismatch. NWs grown on the Si (111) substrate [Figure 5(c)] had a slightly random orientation, also having diameters in the range of $150-200 \mathrm{~nm}$ and lengths from 1-2 $\mu \mathrm{m}$. Finally, the NWs grown on GaN [Figure 5(d)] showed strong vertical orientation, with diameters of 20-40 $\mathrm{nm}$ and lengths of 0.7-1.0 $\mu \mathrm{m}$ [20].

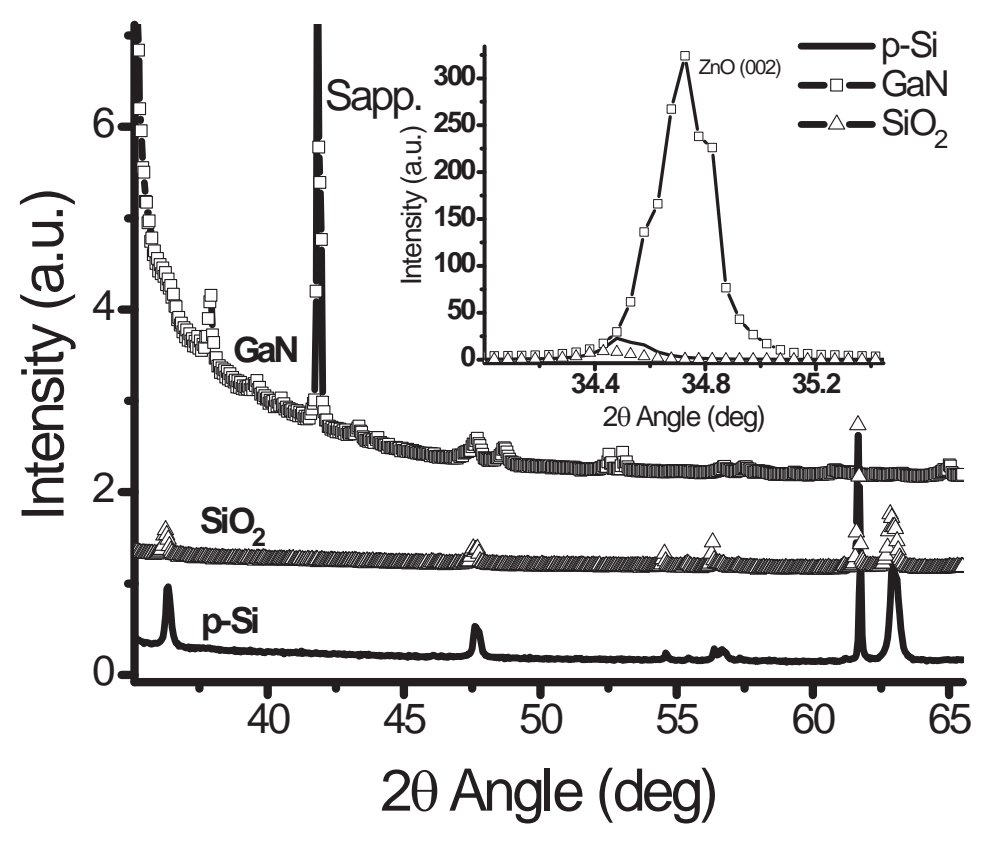

Figure 6. X-ray diffraction (XRD) of ZnO NWs grown using MOCVD on p-Si (solid), GaN/sapphire (square) and $\mathrm{SiO}_{2}$ (triangle). The inset shows the $\mathrm{ZnO}$ peak associated with $\mathrm{ZnO}$ oriented along (002) and GaN [20].

Figure 6 shows the XRD pattern for the $\mathrm{ZnO}$ NWs grown on $\mathrm{p}-\mathrm{Si}, \mathrm{GaN}$, and $\mathrm{SiO}_{2}$ substrates [10]. The inset of Figure 2 shows dominant peaks related to $\mathrm{ZnO}(002)$. The peak at $34^{\circ}(2 \theta)$ for $\mathrm{ZnO}$ grown on $\mathrm{p}-\mathrm{Si}$ and $\mathrm{SiO}_{2}$ substrates incorporated the overlapping of $\mathrm{ZnO} N W s$ (002) and 
$\mathrm{ZnO}$ thin film (002). An additional diffraction peak associated with $\mathrm{GaN}$ was present for the GaN/sapphire substrate. ZnO NWs oriented along the (002) direction had full-widths at half maxima (FWHM) and c-lattice constants of $0.0498(\theta)$ and $5.1982 \AA$ at $34.48^{\circ}(2 \theta)$ for p-Si, $0.0497(\theta)$ and $5.1838 \AA$ at $34.58^{\circ}(2 \theta)$ for $\mathrm{GaN}, 0.0865(\theta)$ and $5.1624^{\circ}$ at $34.38^{\circ}(2 \theta)$ for $\mathrm{SiO}_{2}$, and $0.0830^{\circ}(\theta)$ and $5.2011 \AA$ at $34.46^{\circ}(2 \theta)$ for sapphire.

The quality of the $\mathrm{ZnO}$ epilayers utilized as seed layers to grow $\mathrm{ZnO} \mathrm{NWs}$ was also characterized. The $\mathrm{ZnO}$ thin films were oriented along (002) and had a maximum at $34.58^{\circ}$ with FWHM of $0.0697(\theta)$ for p-Si, maximum of 34.58 ${ }^{\circ}$ with FWHM of $0.0684(\theta)$ for GaN, and maximum of $34.43^{\circ}$ with $\mathrm{FWHM}$ of $0.0557(\theta)$ for $\mathrm{SiO}_{2}$. Additional shallow diffraction peaks were observed for NWs grown on p-Si and $\mathrm{SiO}_{2}$, which are attributed to $\mathrm{ZnO}(100,101,102$ and 110) as can be seen from Figure 6. As shown in Figure 7, for $\mathrm{ZnO}$ NW growth on sapphire major peaks were observed for $\mathrm{ZnO}(002)$ at $34.46^{\circ}(2 \theta)$ and $\mathrm{Al}_{2} \mathrm{O}$ at $37.91^{\circ}(2 \theta)$, with a minor peak for $\mathrm{ZnO}(101)$ at $36.34^{\circ}(2 \theta)$.

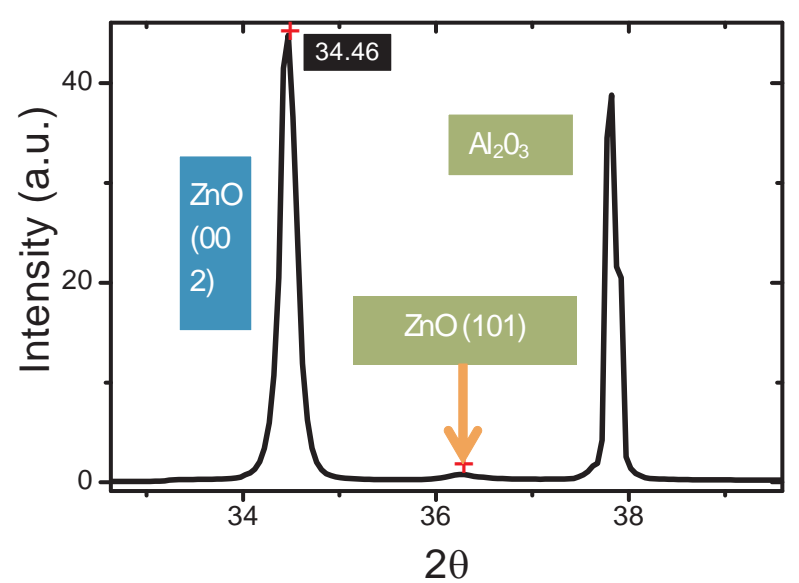

Figure 7. XRD of ZnO NWs grown using MOCVD on sapphire [20]

\subsection{Photoluminescence (PL) measurements}

Figure 8 shows the PL spectra for $\mathrm{ZnO}$ NWs grown on $\mathrm{p}-\mathrm{Si}, \mathrm{GaN}$, and $\mathrm{SiO}_{2}$ substrates [10]. The room temperature PL measurements were performed using a $\sim 280 \mathrm{~nm}$ light source. Single peaks located at $380 \mathrm{~nm}$ having a FWHM of $14.69 \mathrm{~nm}$ and at $378 \mathrm{~nm}$ having a FWHM of $15 \mathrm{~nm}$ were observed for $\mathrm{p}-\mathrm{Si}$ and $\mathrm{SiO}_{2}$ substrates, respectively, corresponding to the recombination of excitons through an exciton-exciton collision process [18-20]. 


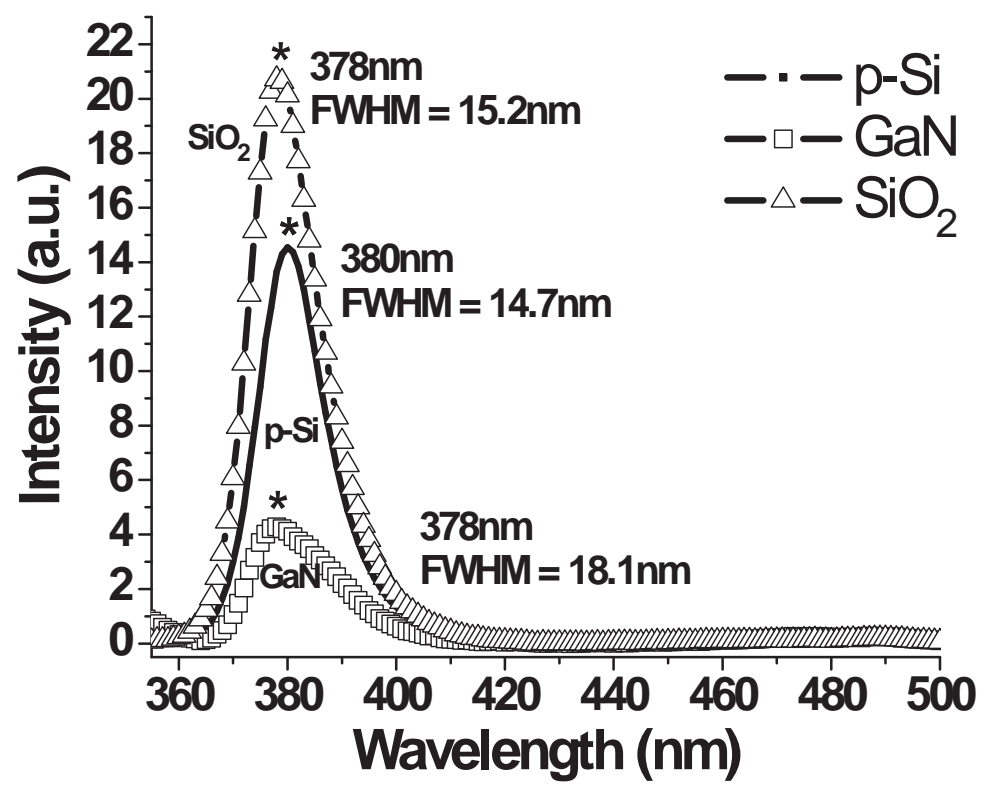

Figure 8. Photoluminescence (PL) of ZnO NWs grown on p-Si (100) (solid) with a single peak at $380 \mathrm{~nm}, \mathrm{GaN}$ (square) with a stronger peak at 378 and $\mathrm{SiO}_{2}$ (triangle) with a single peak at $378 \mathrm{~nm}$ [20].

No defects related to $\mathrm{Zn}$ or $\mathrm{O}$ vacancies were observed, which can be attributed to the confinement of defects at the $\mathrm{ZnO}$ thin film/substrate interface. For the $\mathrm{ZnO}$ NWs grown on $\mathrm{GaN}$, a predominant peak with a FWHM of $18.18 \mathrm{~nm}$ was observed at $378 \mathrm{~nm}$. High stress was evident for $\mathrm{ZnO}$ NWs grown on $\mathrm{GaN}$, which can be observed in Figure 2; this can contribute to the broadening of the peak in comparison to $\mathrm{p}-\mathrm{Si}$ and $\mathrm{SiO}_{2}$. Shallow peaks identified at 474 $\mathrm{nm}$ and $490 \mathrm{~nm}$ through Lorentzian decomposition are attributed to oxygen interstitial and oxygen vacancies, respectively [20].

A UV LED lamp acquired from Sensor Electronic Technology Inc. was used to characterize the UV Photoresponse of the ZnO NW arrays [20]. The lamp comprises eight separate AlGaN based UV LEDs in a TO-3 package spanning the 240-370 nm wavelength range, with a customized power supply capable of independently monitoring and controlling the current of all or any of the LEDs. The Photoresponse was determined by first applying voltage between indium contacts on the front and back sides of a Si NW sample and measuring the resulting current in the dark, and then repeating this procedure while the sample was exposed to radiation from a UV LED at a specific wavelength.

Figure 9 shows the on-off switching characteristics of a $\mathrm{ZnO}$ vertical array NW device when exposed to radiation at $370 \mathrm{~nm}$. This device was found to switch between low and high 


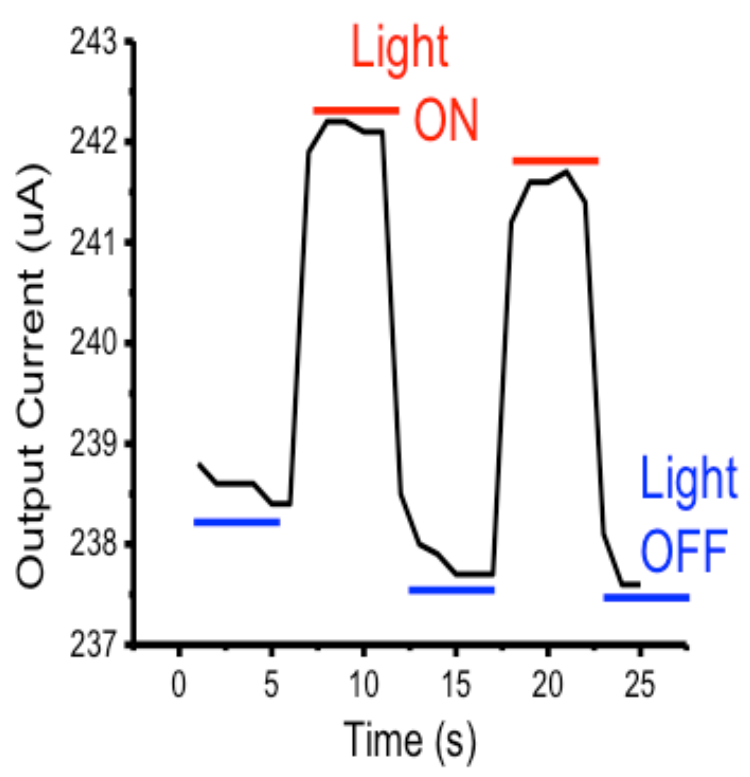

Figure 9. Switching Photoresponse characteristics of ZnO NW device when UV LED source at $\sim 370$ nm turned on and off over approximately 10 s intervals.[20]

conductivity states in approximately $3 \mathrm{~s}$, a faster response than most reported thus far for $\mathrm{ZnO}$ NW based UV detectors.

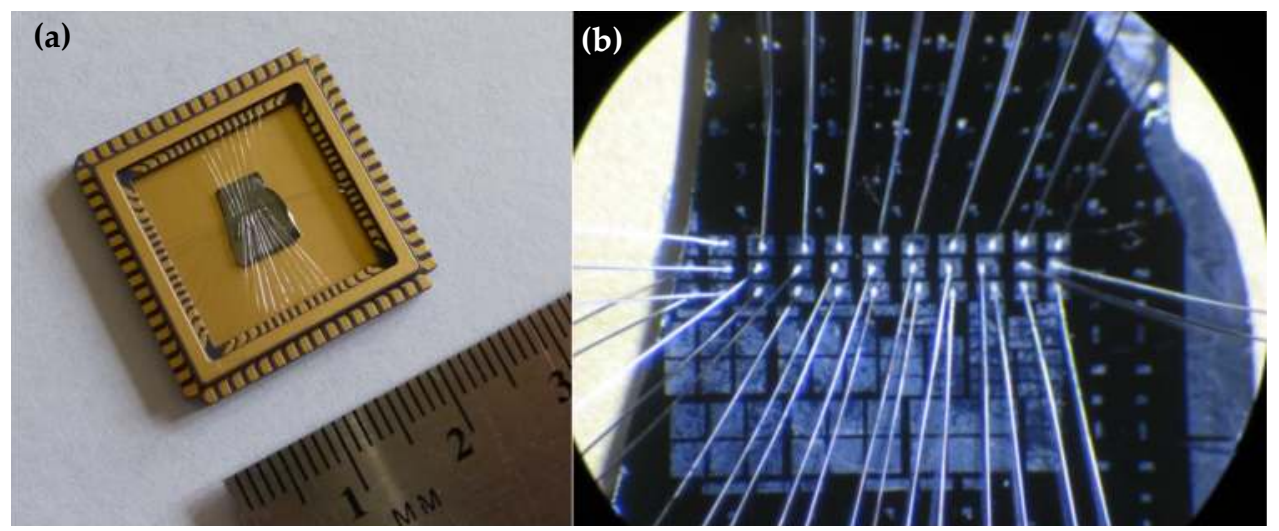

Figure 10. (a) Mounted solar blind NW UV 3x9 pixel array detector device; (b) close-up of device, showing wire bonded pixels [20].

Figure 9(a) shows a mounted and wire bonded NW UV 3x9 pixel array detector device. Incorporation of $\mathrm{Mg}$ allows the detector response to be shifted to shorter wavelengths to 
provide detection in the solar blind region. This device was tested by applying a bias between the top contacts on the pixels, which are apparent in Figure 9(b), and the back contact.

$\mathrm{ZnO}$ nanowires based arrays offer high sensitivity and have potential application in UV imaging systems. ZnO nanowire array based UV detectors have no moving parts, high quantum efficiency, extended lifetimes, low noise, low power requirements, and offer high sensitivity.

$\mathrm{ZnO}$ nanowires have also been evaluated for providing remote power for the stand alone sensors. This type of application has been extensively studied by Professor Z.L. Wang and his team at Georgia Tech [21, 22]. They have shown that $\mathrm{ZnO}$ nanowires can be used as nanogenerators for providing remote power using the Piezo-electric effect. Photovoltaic cells or solar cells are a popular renewable energy technology, relying on approaches such as inorganic p-n junctions, organic thin films, and organic-inorganic heterojunction. However, a solar cell works only under sufficient light illumination, which depends on the location the devices will be deployed, as well as the time of the day and the weather.

Considering that mechanical energy is widely available in our living environment, They have demonstrated [21] the first hybrid cell for concurrently harvesting solar and mechanical energy through simply integrating a dye-sensitized solar cell (DSSC) and a piezoelectric nanogenerator on the two sides of a common substrate. After this, in order to solve the encapsulation problem from liquid electrolyte leakage in the first back-to-back integrated HC, early in 2011, $\mathrm{Xu}$ and Wang improved the prototype design of the HC and developed a compact solid state solar cell. This innovative design convoluted the roles played by the NW array to simultaneously perform their functionality in a nanogenerator and a DSSC. The design and the performance are shown in figure 11 .

Based on these demonstrations of HCs for concurrently harvesting solar and mechanical energies, they have. reported an optical fiber-based three-dimensional (3D) hybrid cell, consisting of a dye-sensitized solar cell for harvesting solar energy and a nanogenerator for harvesting mechanical energy; these are fabricated coaxially around a single fiber as a coreshell structure (Figure 11). The optical fiber, which is flexible and allows remote transmission of light, serves as the substrate for the 3D DSSC for enhancing the electron transport property and the surface area, and making it suitable for solar power generation at remote/concealed locations. The inner layer of the HC is the DSSC portion, which is based on a radically grown ZnO NW array on an optical fiber with ITO as the bottom electrode. The dye-sensitized ZnO NW array was encapsulated by a stainless steel capillary tube with a Pt-coated inner wall as the photo- anode for the DSSC. The stainless steel tube also serves as the bottom electrode for the outer layer of the nanogenerator, with densely packed $\mathrm{ZnO}$ NWs grown on its outer wall.

Another exciting application of $\mathrm{ZnO}$ nanowires is designing, fabricating, and integrating arrays of nanodevices into a functional system are key to transferring nanoscale science into applicable nanotechnology as shown in Figure 12.

Recent work [22] on three-dimensional (3D) circuitry integration of piezotronic transistors based on vertical zinc oxide nanowires as an active taxel-addressable pressure/force sensor matrix for tactile imaging. Using the piezoelectric polarization charges created at a metal- 
semiconductor interface under strain to gate/modulate the transport process of local charge carriers, we designed independently addressable two-terminal transistor arrays, which convert mechanical stimuli applied to the devices into local electronic controlling signals.

The device matrix can achieve shape-adaptive high-resolution tactile imaging and selfpowered, multidimensional active sensing. The 3D piezotronic transistor array may have applications in human-electronics interfacing, smart skin, and micro- and nano-electromechanical systems.

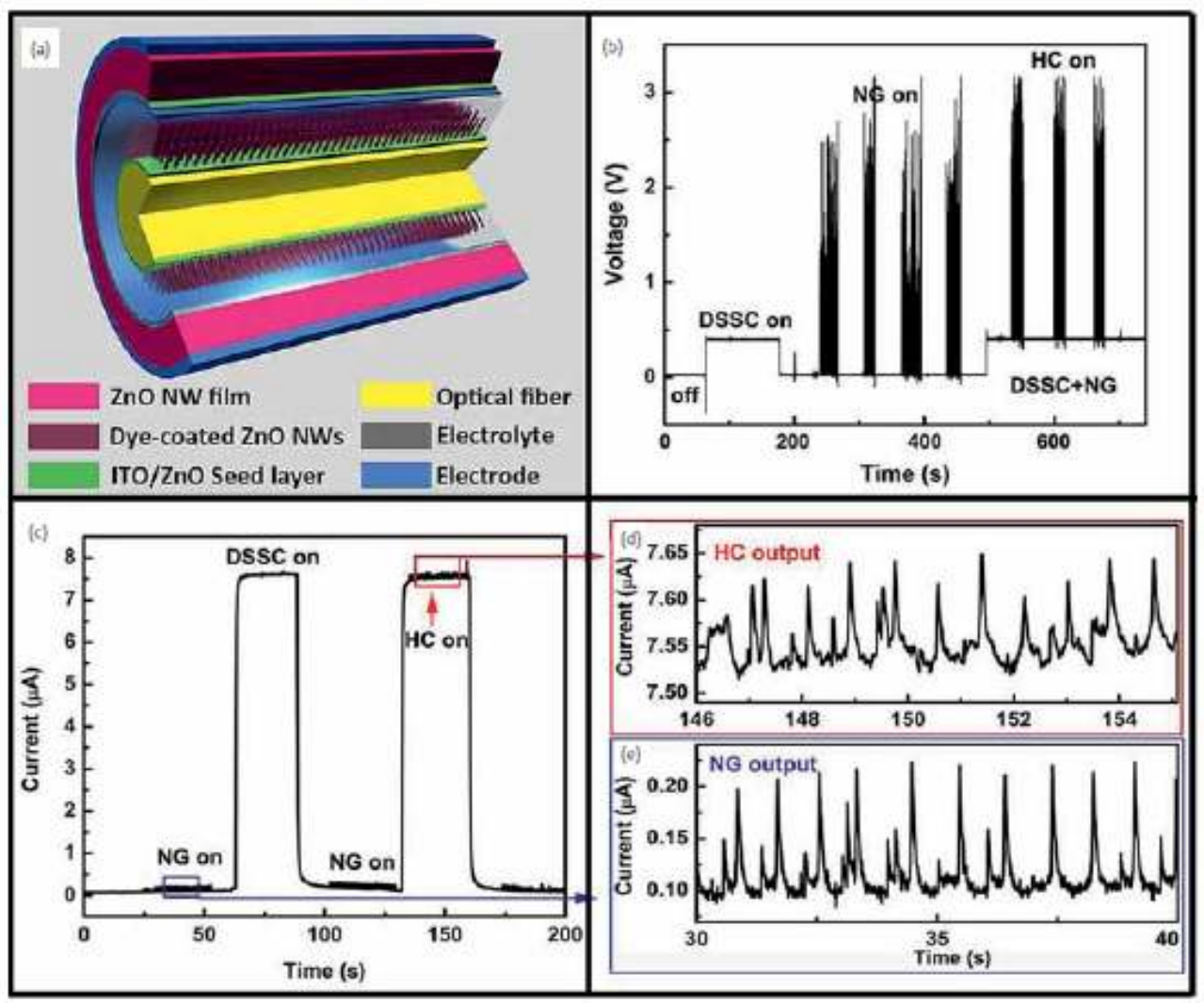

Figure 11. Design and performance of a 3D optical fiber based hybrid cell (HC) consisting of a dye-sensitized solar cell (DSSC) and a nanogenerator (NG) for harvesting solar and mechanical energy. (a) The 3D HC is composed of an optical fiber based DSSC with capillary tube as counter electrode and a NG on top. (b) Open-circuit voltage (VOC) of the HC when the NG and the DSSC are connected in series, where $\operatorname{VOC}(H C)=\operatorname{VOC}(D S S C)+\operatorname{VOC}(N G)$. (c) Short-circuit current (ISC) of the HC when the NG and the DSSC are connected in parallel. (d) and (e) Enlarged view of ISC(HC) and ISC(NG), clearly showing that ISC(NG) is $0.13 \mu \mathrm{A}$, the ISC(DSSC) is $7.52 \mu \mathrm{A}$, and the ISC(HC) is about $7.65 \mu \mathrm{A}$, nearly the sum of the output of the solar cell. [21]. 

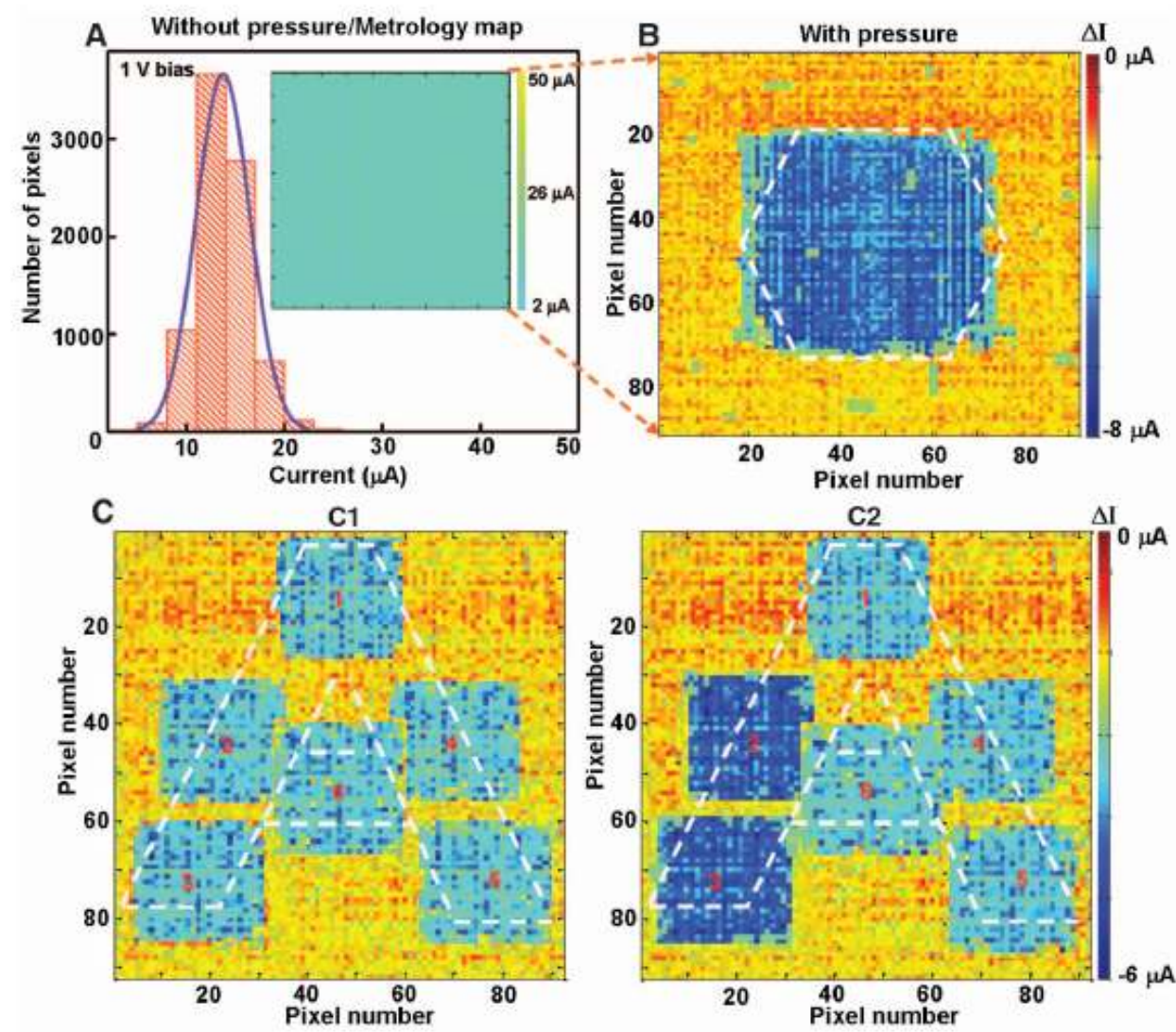

Figure 12. Tactile imaging and multidimensional sensing by the fully integrated $92 \times 92$ SGVPT array. (A) Metrology mapping (inset) and statistical investigation of the fully integrated SGVPT array without applying stress. (B) Current response contour plot illustrating the capability of SGVPT array for imaging the spatial profile of applied stress. Color scale represents the current differences for each taxel before and after applying the normal stress. The physical shape of the applied stress is highlighted by the white dashed lines. (C) Multidimensional sensing by an SGVPT array exhibits the potential of realizing applications such as personal signature recognition with maximum security and unique identity. The shape of a "written" letter A is highlighted by the white dashed lines. [22].

\section{Development of GaN UVAPD for ultraviolet sensor applications}

High resolution imaging in UV bans has a lot of applications in Defense and Commercial applications. The shortest wavelength is desired for spatial resolution which allows for small pixels and large formats. UVAPD's have been demonstrated as discrete devices demonstrating gain. The next frontier is to develop UV APD arrays with high gain to demonstrate high resolution imaging. We will discuss model that can predict sensor performance in the UV band 
using APD's with various gain and other parameters for a desired UV band of interest. SNR's can be modeled from illuminated targets at various distances with high resolution under standard atmospheres in the UV band and the solar blind region using detector arrays with unity gain and with high gain APD's [23-26].

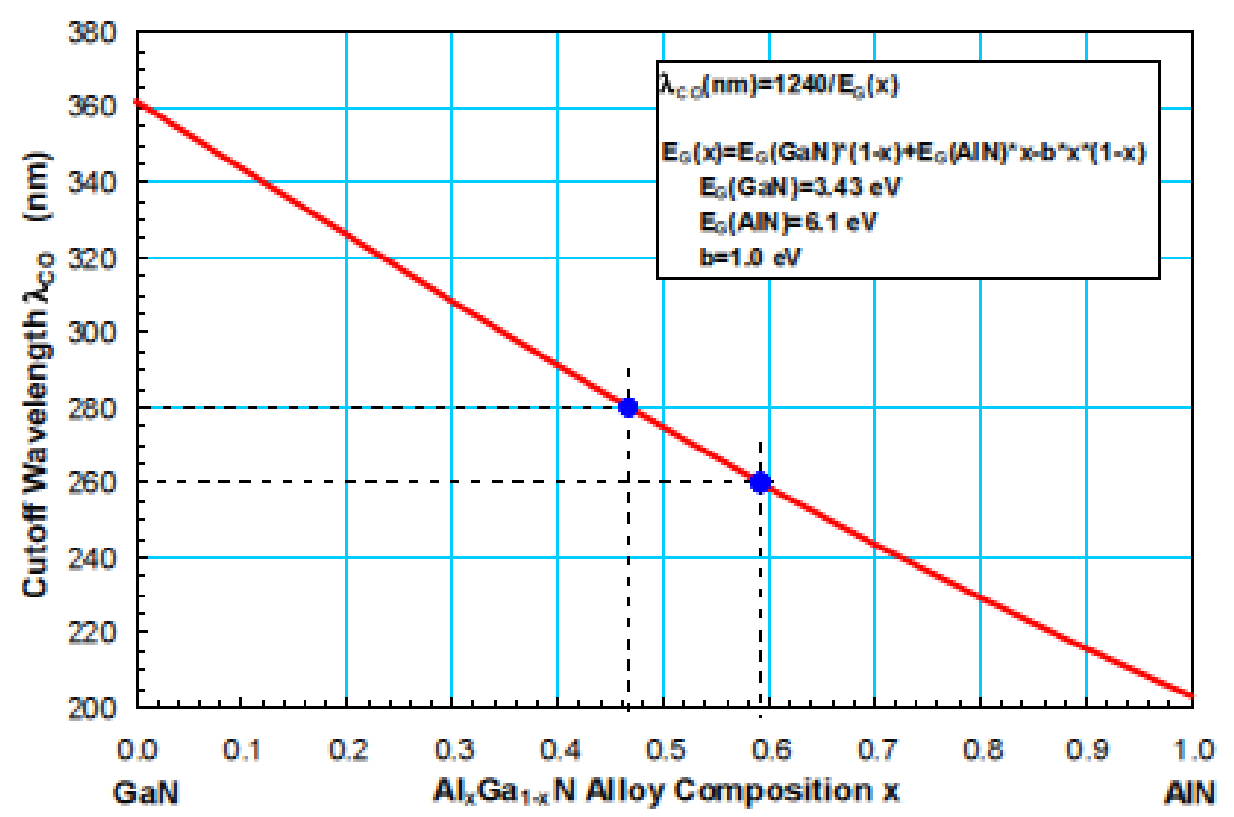

Figure 13. Relationship between alloy composition of AIGaN and the corresponding spectral cutoff for the UV detector arrays [23].

Figure 13 presents the relationship between the alloy composition of Gallium and Aluminum in $\mathrm{Al}_{x} \mathrm{Ga}_{1-\mathrm{x}} \mathrm{N}$ that determines the cut-off wavelength of the UV detector for $\mathrm{p}-\mathrm{i}-\mathrm{n}$ [23-24] and also for UV APD's. Deep Ultra Violet (DUV) will require addition of larger composition of Aluminum in $\mathrm{Al}_{x} \mathrm{Ga}_{1-\mathrm{x}}$ N. [25].

\section{GaN /AlGaN UV APD growth}

Figure 14 presents the High-Temperature MOCVD system by Aixtron. This new reactor design and capability has the ability to grow high quality GaN and AlGaN material with doping for GaN/AlGaN UVAPD applications [26].

Figure 15 presents the device structure of a back-side illuminated AlGaN UV APD. The substrate in this device structure is double side polished AlN substrate. The use of AlN substrate allows the UV APD device structure to be back-side illuminated and can be inte- 
grated with silicon CMOS electronics. Figure 16 presents the Reciprocal Space mapping of AlGaN on AlN substrate and Sapphire substrate. The data for sapphire substrate shows increased strain and mosaicity compared with AlN substrate.
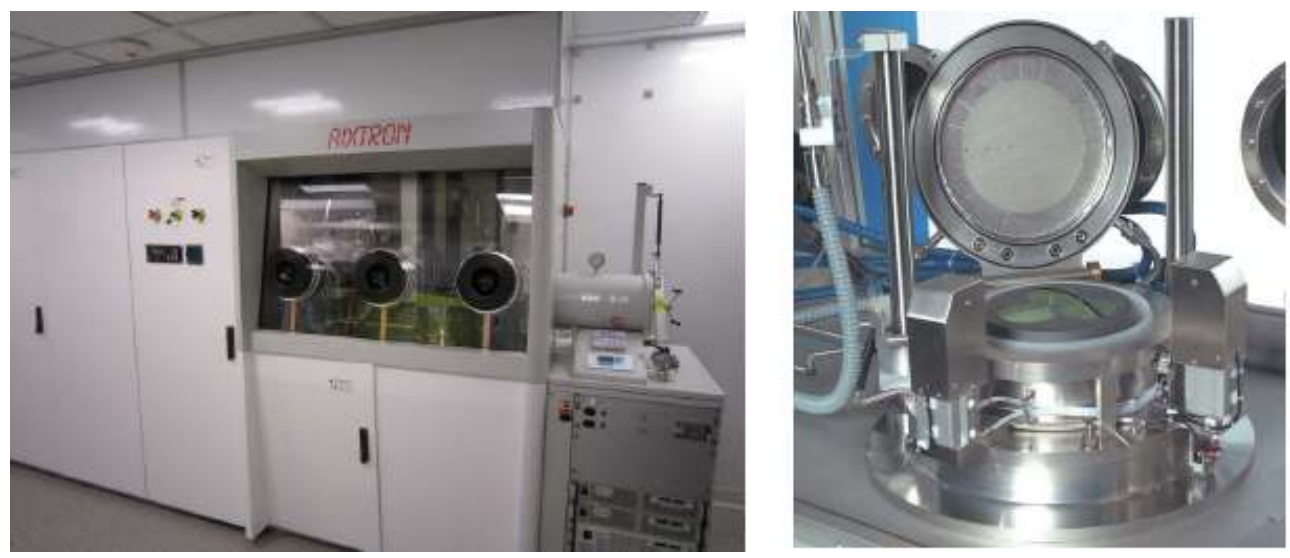

Figure 14. Photograph of New-generation AIXTRON CCS $3 \times 2$ " high temperature III-Nitride $3 \times 2$ MOCVD growth Chamber open for loading wafers showing close-coupled showerhead [26]

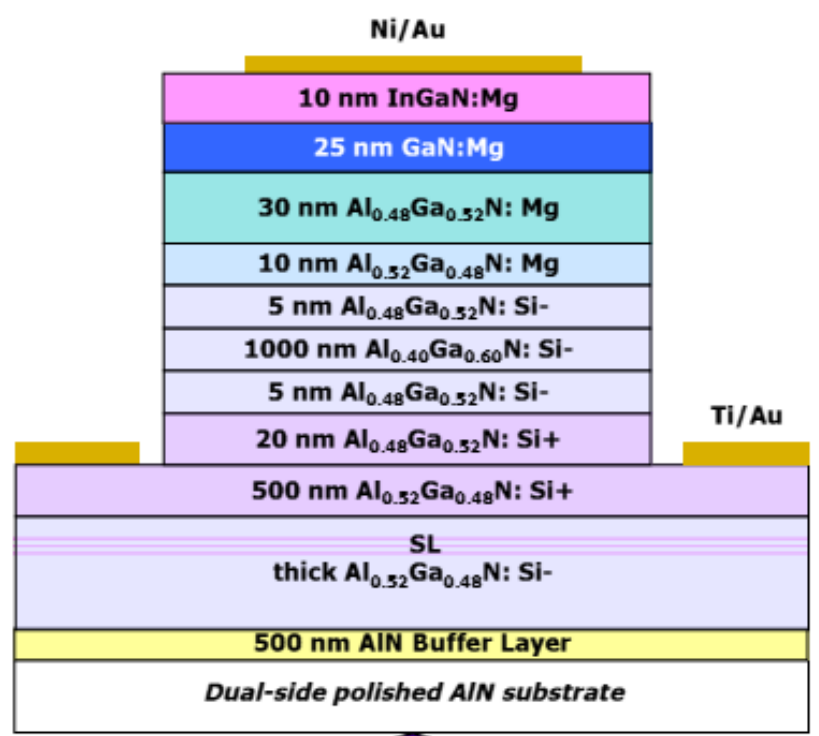

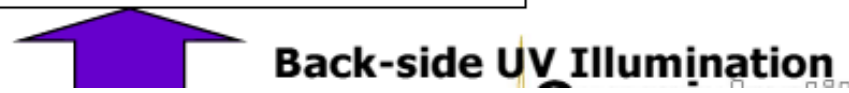

Figure 15. Device Structure Cross-section of prototype Back-Side Illuminated AIGaN UV APD [27] 


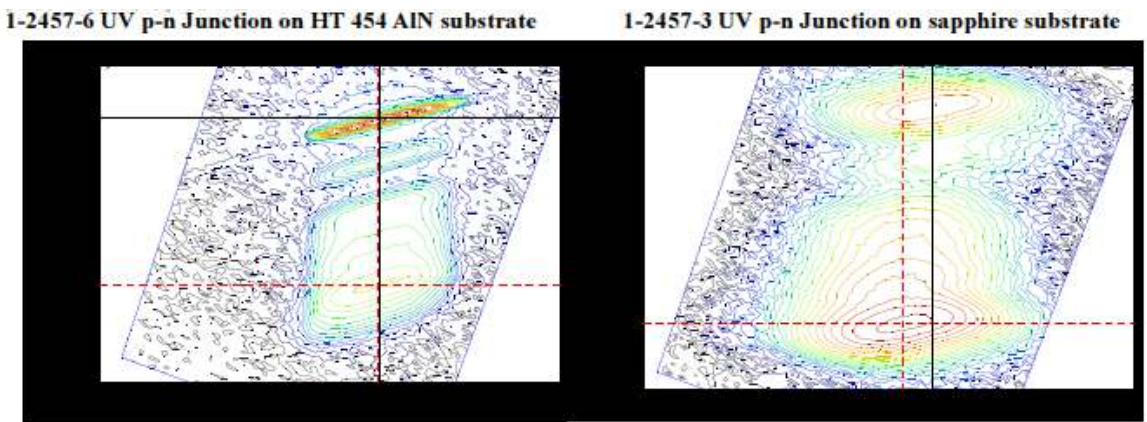

Figure 16. Reciprocal Space mapping of AIGaN p-n junctions on AIN and Sapphire Substrates [28]
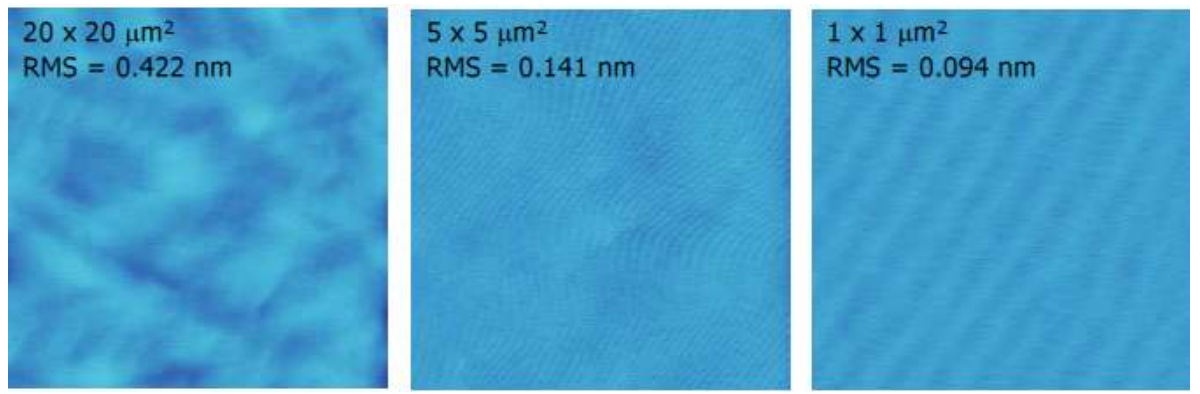

Figure 17. Microscopic surface morphology using AFM on GaN p-i-n structure grown on GaN/Sapphire template. No specific surface defects are observed [27]

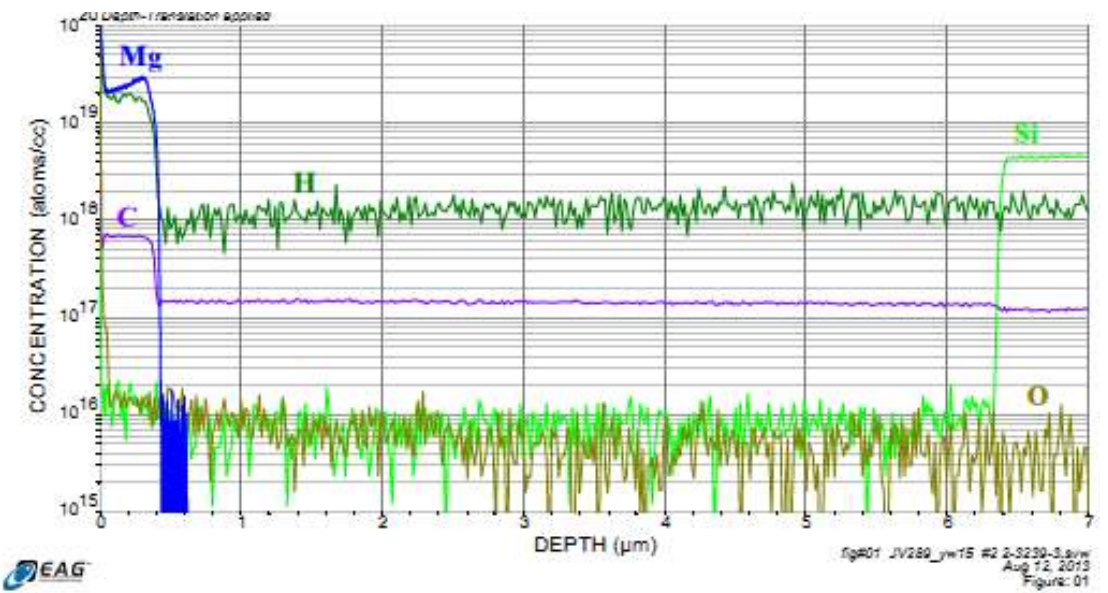

Figure 18. SIMS analysis of GaN p-i-n structure on GaN/Sapphire template, the data shows controlled Si and Mg doping for $\mathrm{n}$ - and p-type layers. The data shows low background doping concentration in GaN layer [28] 
Figure 17 presents the microscopic surface morphology using AFM on GaN p-i-n structure grown on GaN/Sapphire template. No surface defects are observed. These results are encouraging to develop a low cost back-side illuminated UV APD detector array.
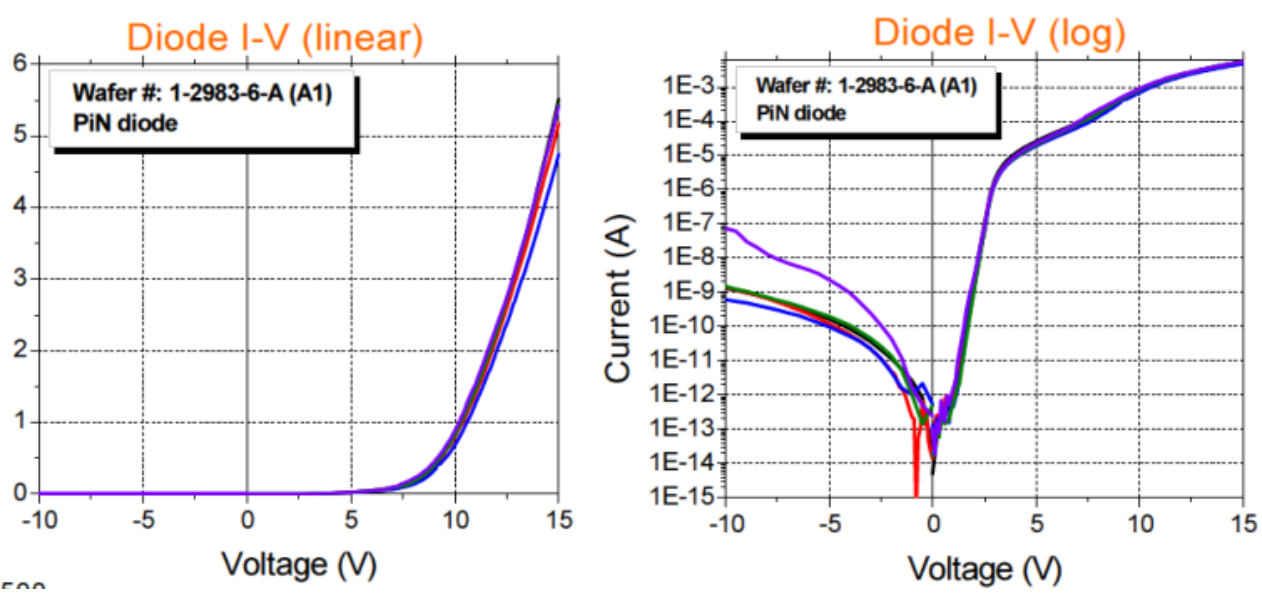

Figure 19. Current - Voltage characteristics of AIGaN UV APD Unpassivated test structure. Further reduction in the dark current will be expected with surface passivation [29].

Figure 18 presents the SIMS analysis of GaN p-i-n structure on GaN/Sapphire template, the data shows controlled $\mathrm{Si}$ and $\mathrm{Mg}$ doping for $\mathrm{n}$ - and p-type layers. The data shows low background doping concentration in GaN layer. The Mg doping is being increased for better p-type conductivity

Figure 19 presents the Current-Voltage characteristics of AlGaNUV APD with spectral response of $300 \mathrm{~nm}$. Further reduction in the dark current will be expected with surface passivation. The future effort is underway to improve the growth characteristics Low-defect-density in substrates and high-quality epitaxial growth technologies are the keys to the successful implementation of a robust high-performance APDs for UV focal plane arrays [29-33].

\section{Visible-Near Infrared (NIR) detector technologies}

The Visible-Near Infrared band $(0.4-1.7 \mu \mathrm{m})$ bridges the spectral gap between the visible and thermal bands in the electromagnetic spectrum. In this spectral band, the primary phenomenology of interest is the reflectance signature of the target, manifested as either its variations in brightness or spectral reflectance, or both.

Infrared imaging in the NIR /SWIR band offers several advantages: it can detect reflected light, offering more intuitive, visible-like images; is better suited for imaging in adverse environments and weather conditions, including fog, dust, and smoke; can also see in low light 
conditions, and use eye safe $1550 \mathrm{~nm}$ illumination; and can generate digital video outputs and thus offering a more dynamic range than traditional image intensifier night vision equipment. Under low light conditions, the sensitivity of the focal plane array is ultimately determined by the $\mathrm{R}_{0} \mathrm{~A}$ product of the photodiode.[ 34-36].

\section{1. $\mathrm{Si}_{1-\mathrm{x}} \mathrm{Ge}_{\mathrm{x}}(\mathrm{SiGe})$ detector arrays}

Like the other two alloy semiconductors mentioned above, SiGe is another example of material that can be used for the fabrication of IR detectors. The key attractive feature of SiGe IR detectors is that they can be fabricated on large diameter Si substrates with size as large as 12inch diameter using standard integrated circuit processing techniques. Furthermore, the SiGe detectors can be directly integrated onto low noise Si ROICs to yield low cost and highly uniform IR FPAs.

Some of the earlier attempts in developing SiGe IR detectors focused on their LWIR applications [34-36]. Renewed efforts are now developing these detectors for application in the NIRSWIR band [36]. For the SiGe material to respond to the SWIR band, its cutoff wavelength is tuned by adjusting the SiGe alloy composition. Si and Ge have the same crystallographic structure and both materials can be alloyed with various Ge concentration. The lattice constant of $\mathrm{Ge}$ is $4.18 \%$ larger than that of $\mathrm{Si}$, and for a $\mathrm{Si}_{1-x} \mathrm{Ge}_{\mathrm{x}}$ alloy the lattice constant does not exactly follow Vegard's law. The relative change of the lattice constant is given by [36]:

$\mathrm{a}_{\text {Si1-x Gex }}=0.5431+0.01992 \mathrm{x}+0.0002733 \mathrm{x}^{2}(\mathrm{~nm})$.

For a $\mathrm{Si}_{1-\mathrm{x}} \mathrm{Ge}_{\mathrm{x}}$ layer with $\mathrm{x}>0$ on a $\mathrm{Si}$ substrate means that the layer is under compressive stress. A perfect epitaxial growth of such a strained heteroepitaxial layer can be achieved as long as its thickness does not exceed a critical thickness for stability. Beyond the critical thickness, the strain is relaxed through the formation of misfit dislocations which can cause an increase in the dark current.

Several approaches have been proposed to reduce the dark current in SiGe detector arrays by several orders of magnitude; these include Superlattice, Quantum dot and Buried junction designs [36-38]. Furthermore, some of these approaches have the potential of extending the wavelength of operation beyond 1.8-2.0 microns. The challenge is to take advantage of these innovative device designs and reduce the dark currents to $1-10 \mathrm{nA} \mathrm{cm}{ }^{-2}$. Figure 20 presents the $\mathrm{SiGe} / \mathrm{Ge}$ detector array using buried junction approach to reduce the surface states and leakage current [36].

Figure 22 shows the Strained-Layer Superlattice (SLS) structure being evaluated for longer detector array response to 2 microns.. It consists of SiGe quantum wells and Si barrier layers, grown on p-type (001) Si substrates. Super lattices having differing Si barrier and Ge well thicknesses to control the strain are grown to optimize wavelength response and dark current.

The SiGe well thicknesses are kept below the critical layer thickness for dislocation formation. To complete the structure, the undoped superlattice is capped with a thin n+ Si cap layer to form the p-n junction. After growth the devices are patterned with a top contact, mesas are etched to provide isolation and the substrate contact is formed. The etched mesa can also be 
passivated to minimize surface recombination as indicated in Figure 22. The device shown in the figure 22 uses substrate illumination, as is needed for use in FPA arrays, and short wavelength response can be improved by thinning the Si substrate.

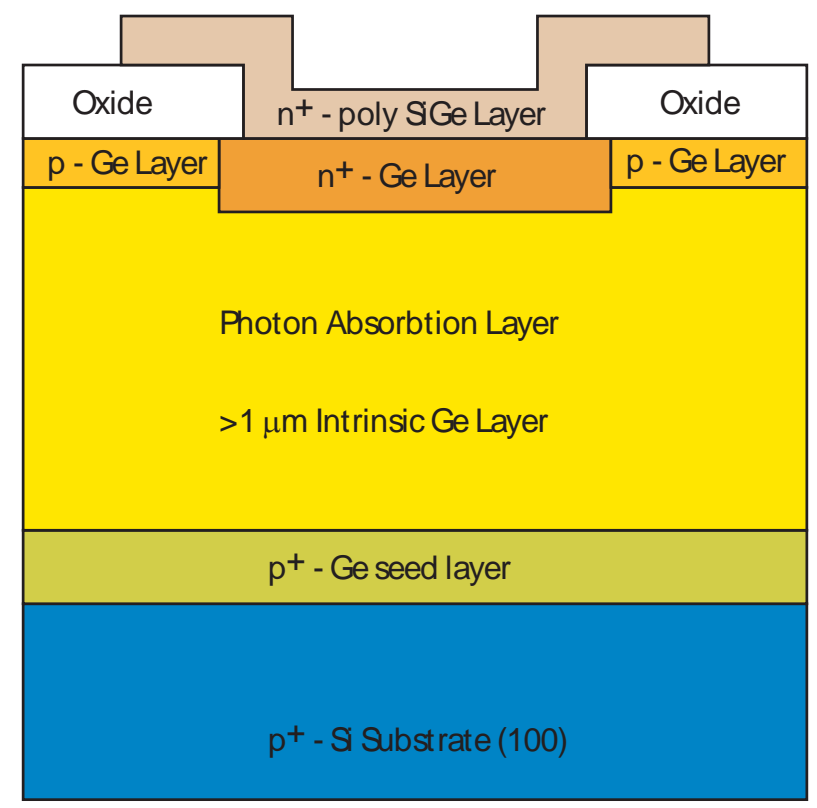

Figure 20. SiGe/Si based buried junction approach to be evaluated for reduced surface states and leakage current [36]
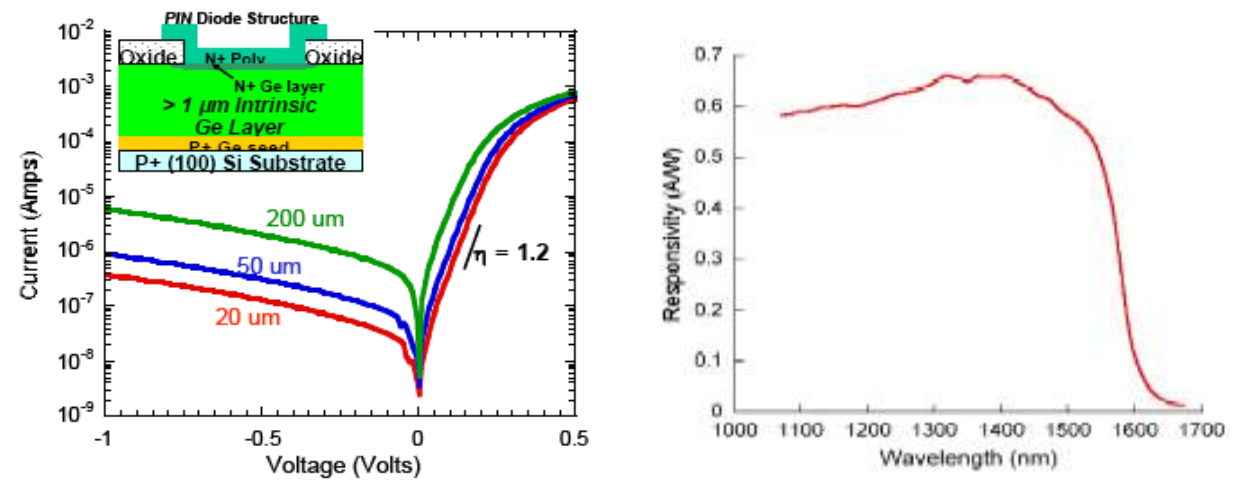

Figure 21. Measured room temperature I-V characteristics for large area diodes with 20, 50 and 200 micron unit cell. The inset shows the schematic device cross section. The spectral response data for SiGe detector is also presented. [38] 


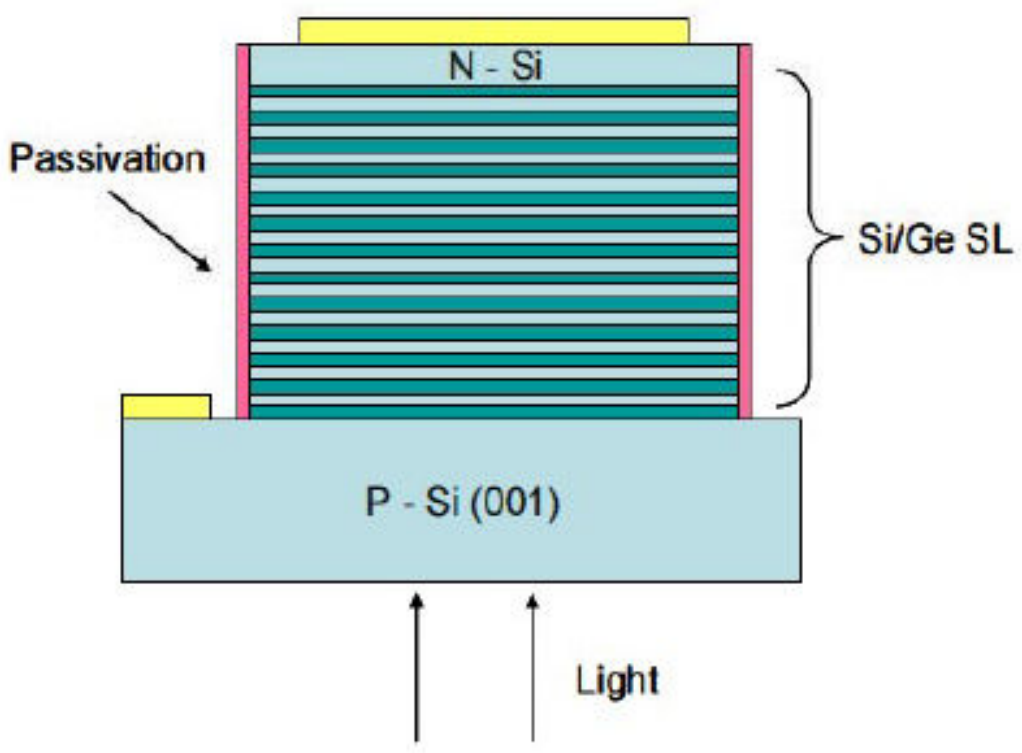

Figure 22. Schematic of detector array structure consisting of a SiGe /Si strained layer Superlattice grown on (001) silicon [38].
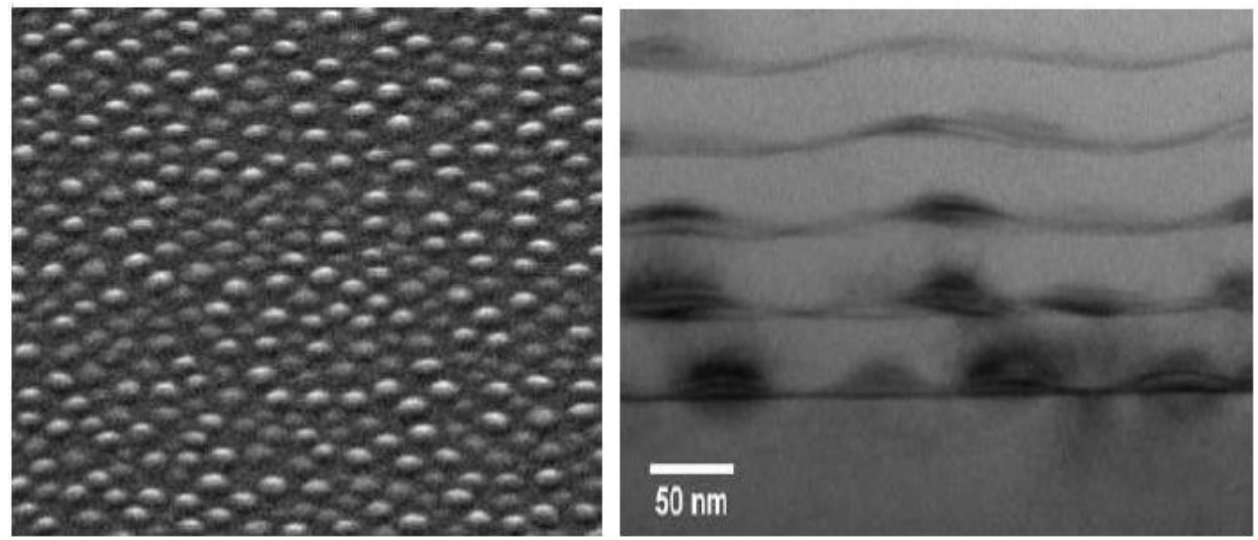

Figure 23. SEM image ( $45^{\circ}$ tilt) of a Ge QD layer deposited on Si. The QDs are $\sim 60 \mathrm{~nm}$ in diameter with a density of $1020 \mathrm{~cm} 2$. Also shown is a Cross-sectional TEM image of Ge/Si QDSL grown. Ge QDs appear with dark contrast compared to Si barriers. [38]. 
The strained-layer superlattice and quantum dot superlattice (QDSL) in the SiGe material system have the potential of developing Vis-NIR detector arrays with longer cutoff wavelength and potentially lower dark current. The advantage of quantum dots is the potential to exploit the optical properties of Ge while avoiding dislocation formation. Ge QDs grown on Si in Stranski-Krastanov mode can be deposited well beyond the critical thickness without dislocation nucleation [39].

Figure 23 shows an SEM image of an array of Ge nanodots grown by MOCVD. These dots are typically $50-75 \mathrm{~nm}$ in diameter with area coverage of $\sim 20 \%$. To increase optical absorption and sensitivity, MOCVD-based growth techniques is being developed for the deposition of Ge/Si quantum dot superlattices (QDSLs), where Ge QDs are alternated with thin $(10-30 \mathrm{~nm}) \mathrm{Si}$ barrier layers. A cross-sectional TEM image of QDSLs is shown in Figure 23.b.

\section{SWIR detector technologies}

The SWIR band $(0.9-2.5 \mu \mathrm{m})$ bridges the spectral gap between the visible and thermal bands in the electromagnetic spectrum. In this spectral band, the primary phenomenology of interest is the reflectance signature of the target, manifested as either its variations in brightness or spectral reflectance, or both.

Infrared imaging in the SWIR band offers several advantages: it can detect reflected light, offering more intuitive, visible-like images; is better suited for imaging in adverse environments and weather conditions, including fog, dust, and smoke; can also see in low light conditions, and use eye safe $1550 \mathrm{~nm}$ illumination that is totally undetectable by regular night vision equipment; and can generate digital video outputs and thus offering a more dynamic range than traditional image intensifier night vision equipment. Under low light conditions, the sensitivity of the focal plane array is ultimately determined by the $\mathrm{R}_{0} \mathrm{~A}$ product of the photodiode.

\section{1. $\operatorname{In}_{\mathrm{x}} \mathrm{Ga}_{1-\mathrm{x}} \mathrm{As}$ detector array development}

For SWIR imaging, InGaAs is one of the widely used detector materials due to its low dark current. The detector material can be prepared using any of the following techniques: Molecular beam epitaxy (MBE), metal-organic chemical vapor deposition (MOCVD), liquid phase epitaxy (LPE), hydride-transport vapor phase epitaxy (VPE), and atomic layer epitaxy (ALE). InGaAs layers are typically grown on lattice matched InP substrates using an alloy composition of $x=0.53[40-42]$.

The spectral response typically covers $0.9-1.7 \mu \mathrm{m}$ at room temperature. By increasing the composition to $\mathrm{x}=0.82$, InGaAs is able to extend its cutoff to $2.6 \mu \mathrm{m}$. However, the crystal defects due to epitaxy and the decreased shunt resistance, due to a smaller band gap, degrade performance at the longer cutoff wavelengths. [43].

The band gap [7-1] of the strained $\operatorname{In}_{x} \mathrm{Ga}_{1-x}$ As: InP structure can be tailored by varying the alloy composition during crystal growth according to the equation: 
$E_{g}(e V)=\left(E_{g_{\text {GaAs }}}-\frac{\alpha_{\text {GaAs }} T^{2}}{T+\beta_{\text {GaAs }}}+\left(E_{g_{\text {InAs }}}-\frac{\alpha_{\text {InAs }} T^{2}}{T+\beta_{\text {InAs }}}-E_{g_{\text {GaAs }}}+\frac{\alpha_{\text {GaAs }} T^{2}}{T+\beta_{\text {GaAs }}}\right) x-0.475 x(1-x)\right.$

Where $\mathrm{E}_{\mathrm{g}}$ is the band gap in $(\mathrm{eV}), \alpha$ and $\beta$ are fitting parameters, and $\mathrm{x}$ is the In: As ratio. The cut-off wavelength can be calculated from the expression $\lambda_{\mathrm{co}}=\mathrm{hc} / \mathrm{E}_{\mathrm{gap}}$

The response can be extended to include the visible wavelength range by removing the InP substrate. There has been an intensive effort to develop InGaAs arrays for Low Light Level (LLL) SWIR imaging [40-46]. An example is in astrophysical space based observatories that are very demanding on the detectors due to the very low IR flux levels. Such low flux levels represent the detection of few photons over long integration times and, therefore, require extremely low dark current photodiodes hybridized to a high performance ROIC stage. For such LLL applications there are challenges ahead to further lower noise, reduce pixel size, fabricate larger arrays, achieve higher operating temperatures, and reduce production cost.

The spectral response of InGaAs diodes at room temperature is in the $0.9-1.67 \mu \mathrm{m}$ wavelength range which matches with the ambient night glow spectrum. Imaging under such low light conditions requires that the noise of the detector be extremely low. A significant portion of the noise is contributed by the dark current of the InGaAs detector and the readout noise. Dark current consists of unwanted thermally generated carriers that can cause the detector to produce a random varying output signal.

It is associated with interfacial, diffusional, G-R, and tunneling currents. The temperature dependence of the dark current is primarily due to the intrinsic carrier concentration which depends exponentially on the temperature. The dark current of the detector can be reduced through appropriate fabrication processes and device design. The impact of dark current noise as a function of read noise is shown in Figure 5.1, where the curves for different pixel pitch map the dark current noise into an equivalent read noise.

For a given read noise, the required dark current density increases as the pixel pitch is decreased. The challenge is to maintain a low dark current density as the pixel pitch is reduced. Simultaneously, the challenge for the read out circuit is to reduce the read noise. If the limitation is due to the detector and its noise level overwhelms the source signal, the solution may be to use an external illuminator or cool the detector. The choice of either solution will depend on a tradeoff between size, weight, and power requirements ( $\mathrm{SWaP})$.

As mentioned above, the dark current of the detector can be reduced through appropriate fabrication processes and device designs. By focusing on the growth conditions for the InGaAs absorption layer, heterointerfaces and the passivation layer, researchers have been able to demonstrate dark current density below $1.5 \mathrm{nA} / \mathrm{cm} 2$ at $77^{\circ} \mathrm{C}$ for $15 \mu \mathrm{m}$ pitch arrays as shown Figure 24 .

In scaling to small pixel pitch, further effort is continued to develop wafer processing parameters and methods that reduce surface related perimeter effects and enable small pixel pitch InGaAs detectors with dark current densities comparable to large $(25 \mu \mathrm{m})$ pixel pitches 
detectors [5-6]. Figure 26 presents a plot of dark current density, measured at $20^{\circ} \mathrm{C}$, for eight different, $300 \times 10$ pixel test arrays distributed across a 3" wafer. The average dark current density at $-100 \mathrm{mV}$ was $2.95 \mathrm{nA} / \mathrm{cm} 2$.

Further effort is underway to demonstrate large format $(>1 \mathrm{~K} x 1 \mathrm{~K})$ and small pixel $(<20 \mu \mathrm{m})$ InGaAs focal plane arrays (FPAs) for a variety of low light level (LLL) imaging applications such as night vision. These applications demand extremely low detector dark current and Si read-out integration circuit (ROIC) noise [47].

Recent work [47] has demonstrated significant progress in InGaAs detector array development on a 4" wafer as shown in figure 27; and also reducing dark current density for 10-20 $\mu \mathrm{m}$ pixel arrays, (3) developing sub-10 $\mu \mathrm{m}$ pixel array technology and demonstrating the feasibility of making $5 \mu \mathrm{m}$ pixel arrays, and (4) reducing the capacitance of small pixels [47]. Figure 28 demonstrates recent results for spectral quantum efficiency $(\mathrm{QE})$ as a function of wavelength measured on backside illuminated InGaAs photodiodes test array at different temperatures demonstrating Visible-Near IR response with InP substrate removed [47].

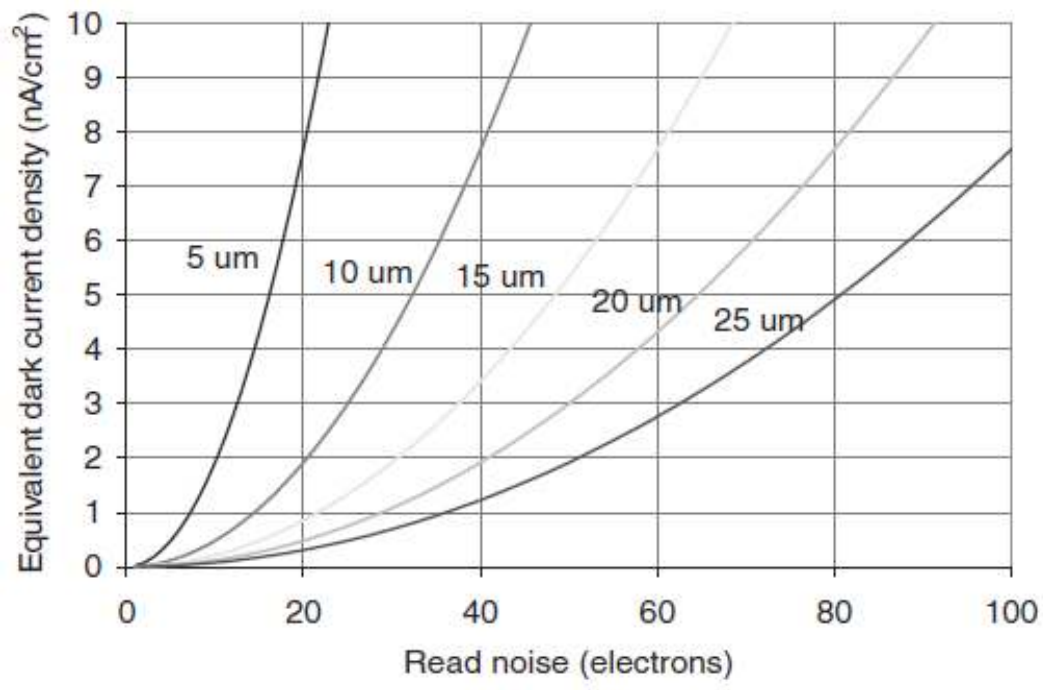

Figure 24. Dark current density versus read noise for different pixel pitches [44]. 


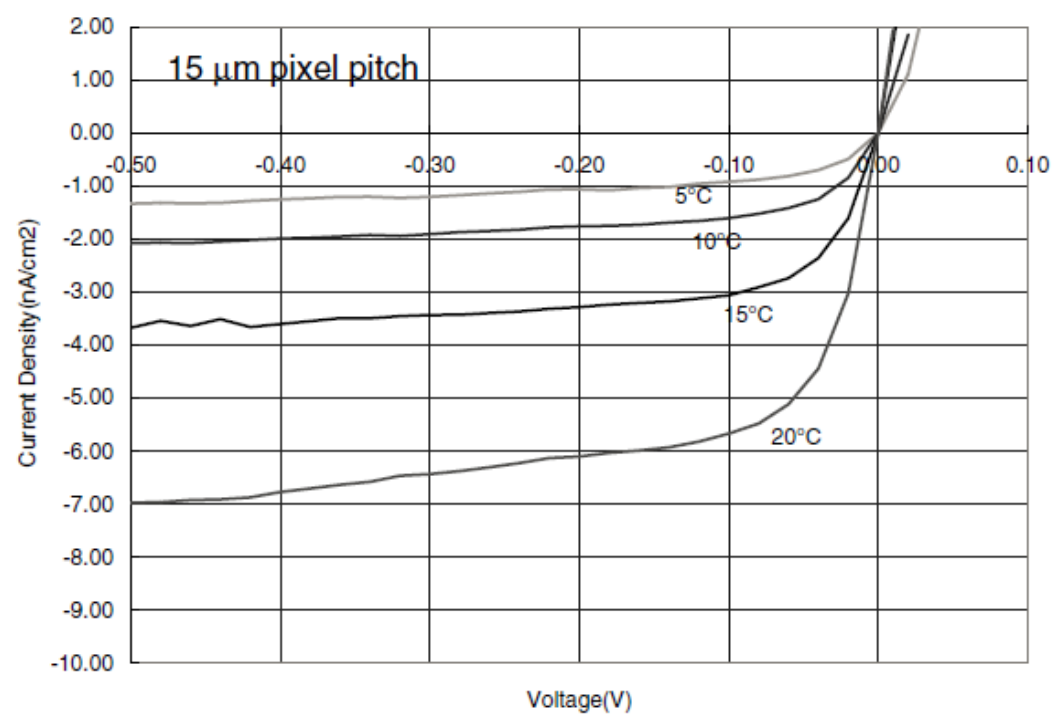

Figure 25. Dark current density at different temperatures using test structures on the wafer. Test arrays have 225 pixels (15 $\mu \mathrm{m}$ pitch) and the guard ring is not biased [45].

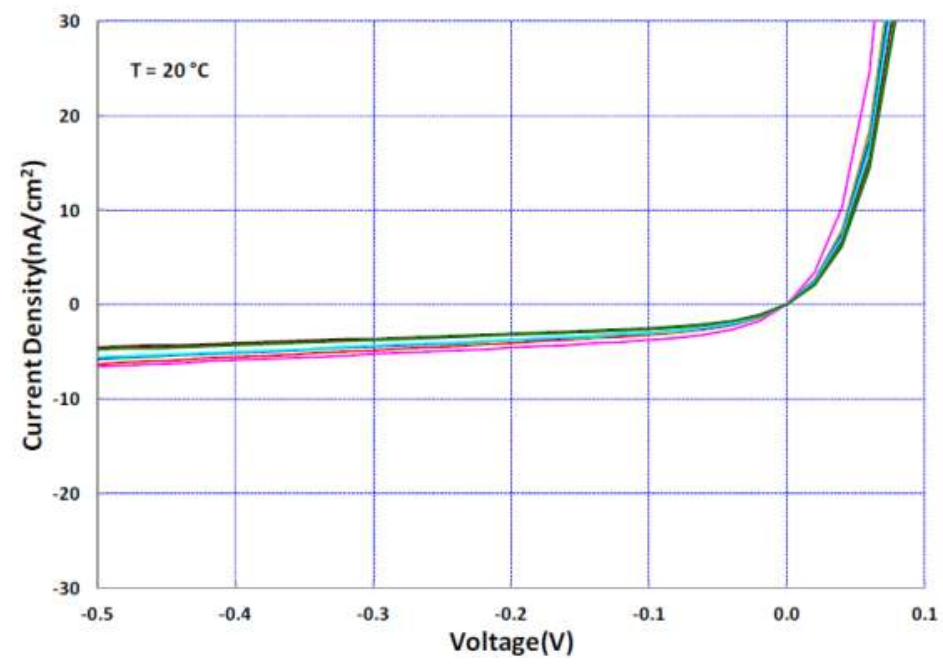

Figure 26. Experimental results for InGaAs test array demonstrating dark current density for eight separate $300 \times 10$, $15 \mu \mathrm{m}$ pitch pixel test arrays measured across a wafer. The average dark current density for the test arrays at $100 \mathrm{mV}$ reverse bias is $2.95 \mathrm{nA} / \mathrm{cm} 2$ at $20^{\circ} \mathrm{C}[45]$. 


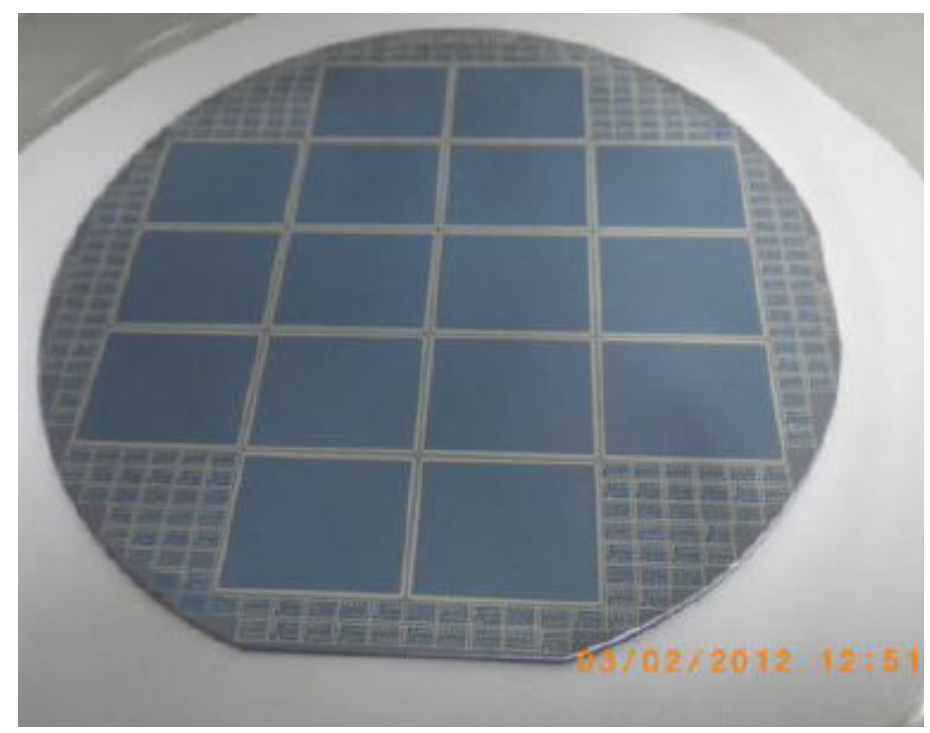

Figure 27. Experimental $1280 \times 1024 / 15 \mu \mathrm{m}$ arrays on 4" wafer surrounded by various test mini-arrays with pitch sizes of $5-20 \mu \mathrm{m}[47]$.

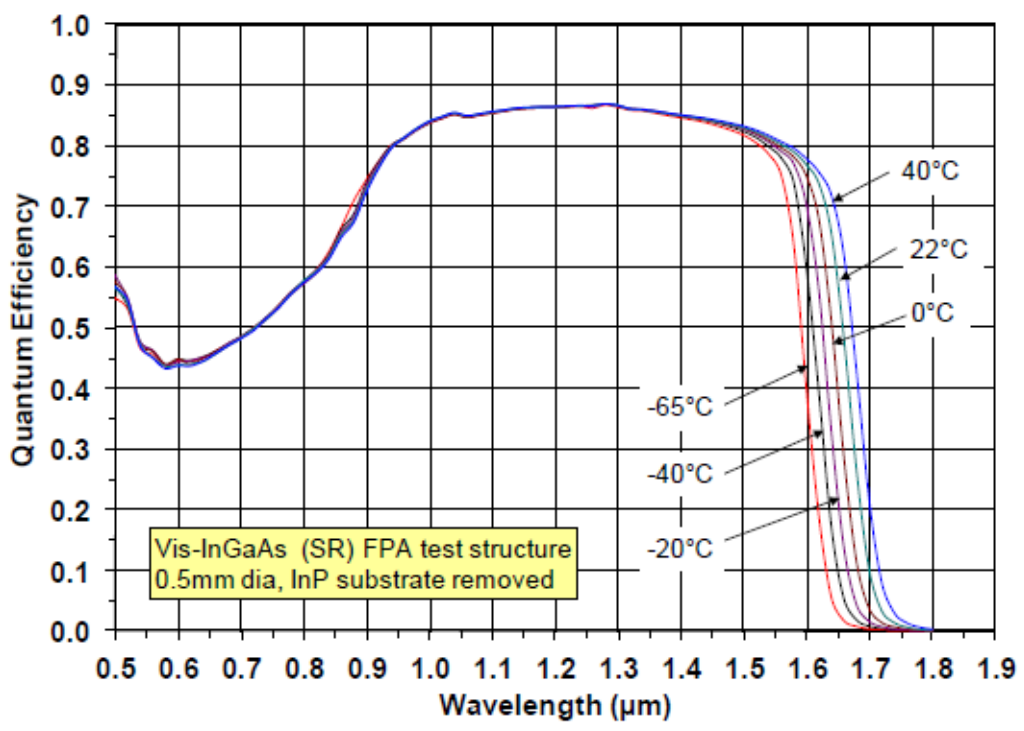

Figure 28. Spectral QE vs. wavelength at different temperatures measured for backside illuminated InGaAs photodiodes test array demonstrating Visible-Near IR response with InP substrate removed [47]. 


\section{Nanostructured detector technology for MWIR and LWIR bands}

EO/IR Sensors and imagers using nanostructure based materials are being developed for a variety of Defense Applications. In this section, we will present recent work under way for development of next generation carbon nanostructure based infrared detectors and arrays. We will discuss detector concepts that will provide next generation high performance, high frame rate, and uncooled nano-bolometer for MWIR and LWIR bands [52-55]. The critical technologies being developed include carbon nanostructure growth, characterization, optical and electronic properties that show the feasibility for IR detection. Experimental results on CNT nanostructures will be presented. Further discussion will be provided for the path forward to demonstrate enhanced IR sensitivity and larger arrays.

The microbolometer based on Si-MEMS device structure has been under development for over 20 years with support from DARPA and the US Army. Two most common Si-MEMS based structures utilize VOx and amorphous silicon based technologies. Several companies such as BAE systems and DRS Technologies are developing and producing 17 micron unit cell 640x 480 and larger arrays using VOx [48-51]. Similarly, L3Communications and other groups are developing and producing 640x480 with 17 micron unit cell using amorphous-Silicon technology [50-51].

We will discuss the use of carbon nanostructures for use as the high performance bolometric element of the MWIR and LWIR bands. As part of this effort, we are exploring development of smaller unit cell bolometer.i.e. 5-10 micron unit cell, with higher TCR and higher frequency response in the 1 to $10 \mathrm{KHz}$ range. The feasibility of such an array can open up a larger number of defense and commercial applications. This section will discuss the efforts under way to explore these possibilities.

\subsection{Design and modeling of CNT-based bolometer characteristics}

To optimize bolometer design, we need to consider several physical key phenomena. From a fundamental point of view, we take the absorbing material to have an extremely large response to infrared radiation. The phonon modes of the material need to be able to easily couple to infrared radiation. Furthermore, once this coupling has been achieved, the absorbed radiation should greatly increase the population of the phonon modes thereby significantly increasing the lattice temperature [52-53].

On a macroscopic scale, this large temperature increase is typically described in terms of a large thermal resistance. The higher the thermal resistance of the material, in general, the higher the resulting temperature will be after absorbing IR. Of course, higher thermal resistance may also give rise to larger thermal noise. This mitigating factor must be balanced with signal response in order to optimize IR sensitivity, or the minimum detectable IR signal.

In summary, for DC operation, we work to maximize the thermal resistance $\mathrm{R}_{\mathrm{th}}$ while achieving an acceptable noise, which maximizes the minimum signal that we can detect. In addition to DC operation, we try to maximize the thermal frequency response of the bolometer. This 
requires that we minimize the thermal capacitance $C_{t h}$ of the device and thereby minimize the thermal $\mathrm{R}_{\mathrm{th}} \mathrm{C}_{\mathrm{th}}$ time constant for the absorbing material.

Temperature Dependent Electrical Characteristics: In addition to considering the DC and transient thermal characteristics of the absorbing material, we need to optimize the electrical response as well. To achieve this, we associate the electrical response with the thermal response by considering the temperature dependent voltage-current characteristics of the electrical material.

A good response is obtained by utilizing a material that has an effectively large variation of electrical resistance with temperature. However, at the same time we want the material to have a temperature coefficient of resistance that is relatively independent of the absolute value of the resistance itself. Therefore, we look at materials that have an exponential relationship between electrical resistance and temperature. For such materials, the Temperature Coefficient of Resistance (TCR) is not strongly dependent on the absolute value of the electrical resistance of the material itself.

\subsubsection{Calculating the thermal response of the CNT bolometer film}

In this work we are assessing the possibility of designing a bolometer using carbon nanotubes as both the IR absorbing material and the electrical response material. Thus, our aim is to first determine the thermal response of the bolometer absorber that is composed of CNTs, and then determine how the electrical characteristics of the CNT material depend on its changes in temperature after IR absorption.

While there are numerous geometries of CNT based material we can consider, for this work we will focus on an absorbing material composed of a CNT film. The film is taken to consist of a random placement of CNTs that is two nanotubes thick.

Heat Flow Equation: To determine the temperature of the material in the presence of IR radiation, we start with the heat flow equation. This is a partial differential equation relating the time rate of change in temperature to the position and the rate of net heat that is absorbed by the material as a function of time and position.

$C v \frac{\partial T}{\partial t}=\kappa \nabla^{2} T+H$

In the equation $C_{v}$ is the thermal capacitance (joules/degree-unit $\mathrm{cm}^{3}$ ) and $\kappa$ is the thermal diffusion coefficient in watts/degree-cm). $H$ is the net power absorbed by the material in watts per unit volume. To solve this equation for the CNT bolometer, we have to first determine $C_{\mathrm{v}}$ and $\kappa$ for the CNT and the CNT film.

Thermal Capacitance of CNT Absorber: To determine the heat capacity of a carbon nanotube, we first determine the internal vibrational energy of the CNT, and then take the derivative with respect to temperature. The internal energy is found by determining the energy of each vibrational mode, multiplying by the probability that the mode is populated using BoseEinstein statistics, and then summing over all of the allowed modes. 
The number of allowed modes will depend on the diameter and wrapping angle of the CNTs present, so we take a statistical sample. Multiplying the individual CNT heat capacity by the number of CNTs in the film provides a reasonable value for the heat capacity of the film. After following this procedure and inserting numerical values for physical constants, we arrive at the following average numerical value for thermal capacity of a CNT $C_{\mathrm{vt}}$, where the length $L$ is in microns and the diameter $d$ is in nanometers

$C_{v t} \sim 1.4 \times 10^{-18}(\mathrm{~L})(\mathrm{d})$

CNT Thermal Diffusion Coefficient: In addition to the thermal capacitance, we need to determine the thermal diffusion coefficient, and eventually the thermal resistance of a single CNT. Experiments on isolated CNTs have fit the coefficient of thermal diffusion to data obtaining the following expression [52-53]:

$\kappa(L, T)=\left\{3.7 \times 10^{-7} T+9.7 \times 10^{-10} T^{2}+\frac{9.3}{T^{2}}\left[1+\frac{0.5}{L}\right]\right\}^{-1}$

From $\kappa$ we can obtain the thermal resistance $R_{\mathrm{T}}$ of a single CNT using the following definition:

$R_{T}=\frac{4 L}{\kappa \pi d^{2}}$

Where $\mathrm{L}, \mathrm{d}$ are the length and diameter of the CNT

Using average values for CNTs gives the following numerical value for the thermal resistance of a CNT

$\mathrm{R}_{\mathrm{T}} \sim 5 \times 10^{8}\left(\mathrm{~L} / \mathrm{d}^{2}\right)$

Where $R_{\mathrm{T}}$ is in units of degrees $\mathrm{K} / \mathrm{Watt}, L$ is in units of microns and $d$ is in units of nanometers. So a tube that is one micron long and one nanometer in diameter will have a thermal resistance $R_{\mathrm{T}}$ of approximately $5 \times 10^{8} \mathrm{~K} / \mathrm{W}$.

Net IR Radiation Power Absorbed: Now that we have the thermal diffusion and capacitance we are almost ready to begin solving the above heat flow equation to determine the temperature of the bolometer. However, before doing so, we need to determine $H$, the net IR power absorbed by the bolometer. We determine this power using the Stefan-Boltzmann Law of blackbody radiation, which relates the net power absorbed to the temperatures of the subject and the bolometer using the following expression:

$H_{n e t}=\sigma A \varepsilon\left(T_{o b j}^{4}-T_{b}^{4}\right)$

Where $H_{n e t} \sigma, A, \varepsilon, T_{o b j}$ and $T_{b}$ are heat absorbed by the bolometer, the Stefan-Boltzmann constant, cross-sectional area, emissivity, object of interest temperature and bolometer absorber temperature, respectively. Figure 1 below shows the net IR power absorbed by the absorber as a function of bolometer temperature for radiating objects at $20^{\circ} \mathrm{C}$ and $36.5^{\circ} \mathrm{C}$. Cooling the bolometer by $30^{\circ} \mathrm{C}$ below room temperature allows for significantly more power to be absorbed, which can give rise to a much stronger signal.

Calculating the Bolometer Temperature Distribution: Using the aforementioned expressions for $\mathrm{C}_{v t} \mathrm{~K}$ and $\mathrm{H}$, we expand on previous work and convert the heat flow equation above into a 


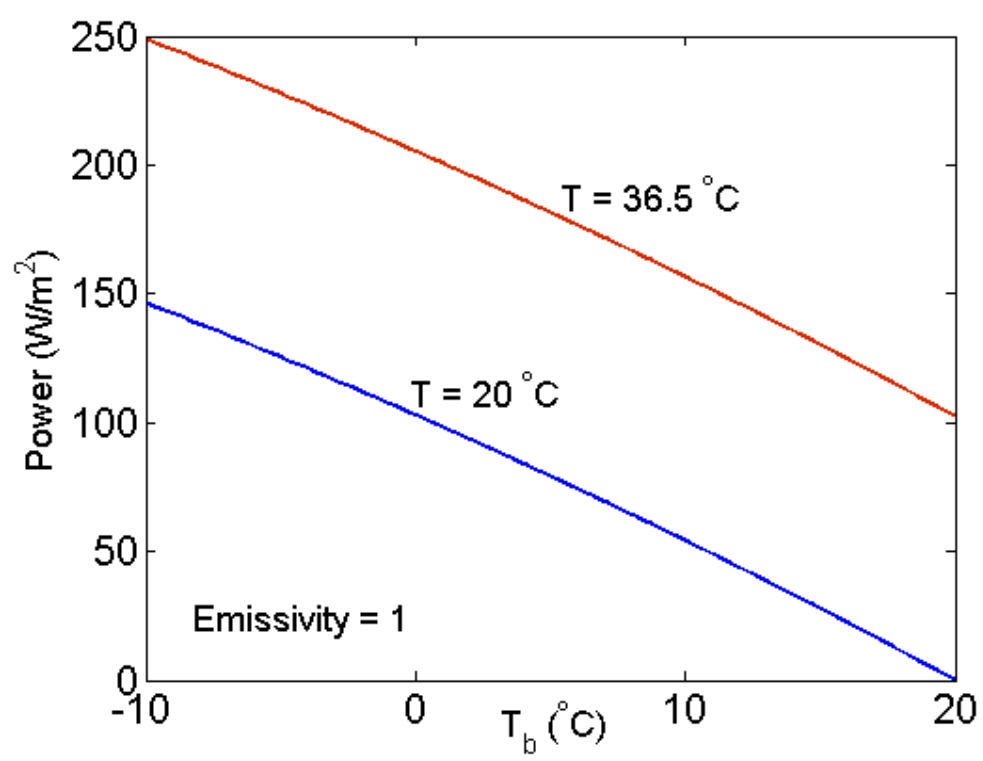

Figure 29. Net power received by bolometer as a function of bolometer temperature.[54]

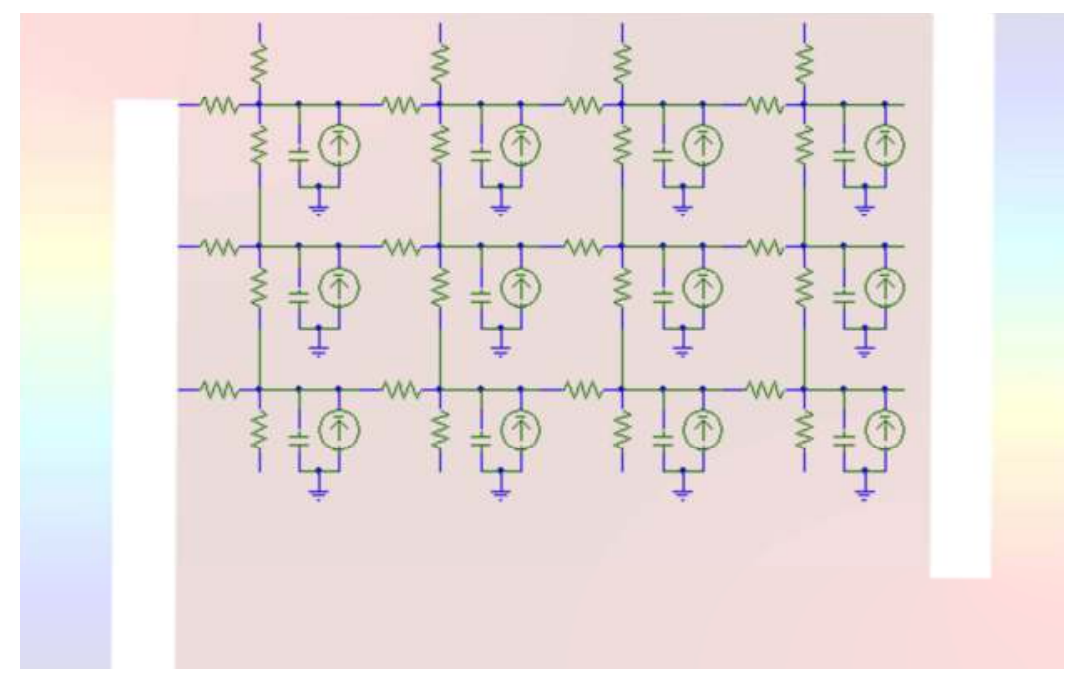

Figure 30. Illustration of thermal network superimposed on bolometer for calculating temperature map of CNT bolometer absorber.[55]

thermal network, illustrated in Figure 2 [6]. In actuality, there are thousands of nodes in the network for which we calculate the temperature for each. 
In Figure 30, each resistor represents the thermal resistance of a CNT in series with the thermal resistance between adjacent CNTs. In addition, the capacitors represent the thermal capacity of a CNT, while the current sources represent the net IR radiation absorbed by each CNT. This thermal network contains thousands of nodes, and there is an equation relating the thermal resistance, capacitance and net power for each node. This system of equations is then solved for the temperature as a function of position and time throughout the bolometer absorber [54].

Results of these calculations for are shown in Figure 31 for different types of CNT networks. Here, we assumed that the net absorbed power is $1 \mathrm{nW}$, and the pixel is tightly packed with the CNTs. The entire pixel's temperature map is obtained with a $100 \times 100$ temperature resolution. For the tubes, we used two different thermal resistance values: $5 \times 10^{8}$ and $1 \times 10^{9} \mathrm{~K} / \mathrm{W}$.

As expected, higher the thermal resistance, higher the temperature difference from the ambient. We note that in general thermal resistance also rises with increasing temperature, resulting in further heating of hot spots compared to the case that this dependency is ignored. The temperature gradient of the contacts legs connecting the film to the readout IC (ROIC) is clearly shown.
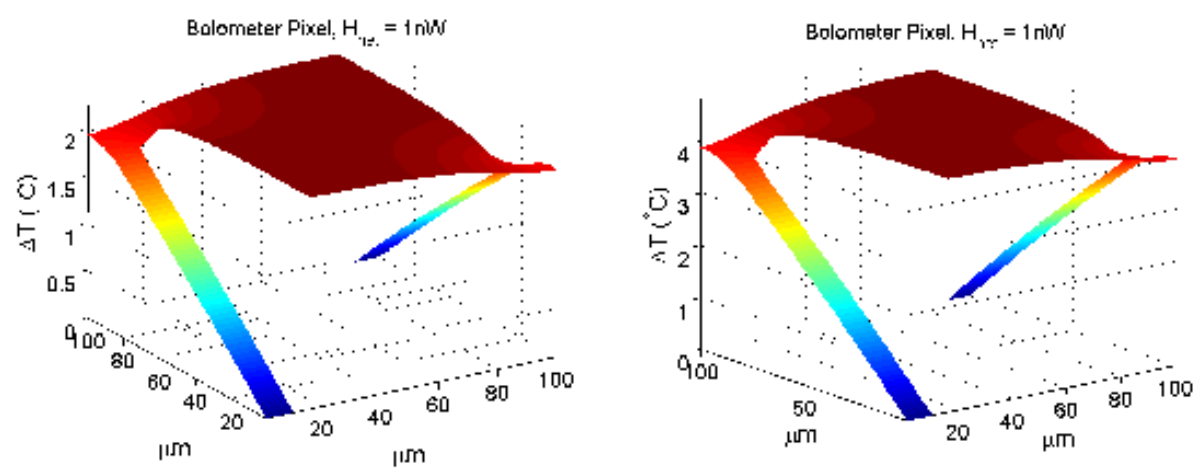

Figure 31. Temperature map of a bolometer pixel when the net absorbed power $H$ is $1 \mathrm{nW}$, and the CNT thermal resistance is $5 \times 10^{8} \mathrm{~K} / \mathrm{W}$ (left) and $1 \times 10^{9} \mathrm{~K} / \mathrm{W}$ (left). We assumed that the pixel is tightly packed with the CNTs. The temperature gradient of the contacts legs connecting the film to the ROIC is clearly shown [54]

\subsubsection{Calculating the electrical response of the CNT film}

To read the temperature that the bolometer pixel reaches after an exposure to infrared radiation, one needs to measure the electrical resistance of the pixel. By comparing this resistance to a look-up table or using the a-priori knowledge of temperature coefficient of 
resistance, the pixel temperature can be determined. Therefore, in addition to having a large thermal resistance which translates into higher temperature rises, a large temperature coefficient of electrical resistance (TCR) is desirable to achieve a higher temperature resolution.

Here TCR is defined as the change in electrical resistance per degree Kelvin divided by the absolute electrical resistance measured at the quiescent point, as follows:

$T C R=\frac{1}{R_{e}} \frac{d R_{e}}{d T}$

Thus, the pixel electrical resistance after it reaches a temperature that is $\Delta \mathrm{T}$ above its ambient becomes $R_{e}(T)=R_{e}(T o)(1+T C R)$. Using this relationship, the pixel temperature is calculated.

To obtain a high temperature resolution, a large change in electrical resistance is needed upon heating. To achieve this, a substantial increase either in electron concentration or velocity (for a given electric field) is necessary. And to this end, materials with junctions where thermionic emission or tunneling are the electrical current bottlenecks offer a good solution. As the tunneling current exponentially rises with temperature, the effective change in their electrical resistance due to temperature becomes large compared to those observed in bulk materials where the change is proportional $T^{\gamma}$ and $\gamma$ is generally $<2$.

Here, a film of CNTs is proposed as the bolometer pixel material, since it is likely to have large thermal resistance and TCR values simultaneously. Both of these favorable properties are partially owed to the junctions between the tubes. As the electrical current flows along the mat, it needs to jump from one tube to the next where they intersect.

At this intersection, the carriers see a potential barrier that they need to tunnel through which gives rise to exponential increase in current upon heating. Assuming that the electron transport across this barrier is governed by a Fowler-Nordheim-type tunneling or thermionic emission, the expected TCR values can be calculated using the following expression:

$I=q \int_{0}^{\infty} T_{t}(E) v(E) \operatorname{DOS}(E) f(E, T) d E$

Where $T_{t}, v, D O S$ and $f$, are the transmission coefficient, thermal velocity, density of states and distribution function, respectively for electrons in the CNT. We perform this calculation a function of barrier height and electric field. The results are shown in figure 4 . The figure on the left shows a contour plot of the theoretical values of the TCR for a CNT film.

The TCR is plotted as a function of electric field between the tubes and the barrier height. Theoretical calculations predict an extremely large TCR, which can be attributed to the relatively large barrier height between adjacent CNTs. If a lower barrier height is assumed, on the order of $0.06 \mathrm{eV}$, then a TCR of approximately $2.5 \%$ is obtained. It is also worth pointing out that such a large TCR comes at the price of extremely low output currents. The bolometer current densities, as a function of barrier height and electric field are shown in Figure 32, on the right. 

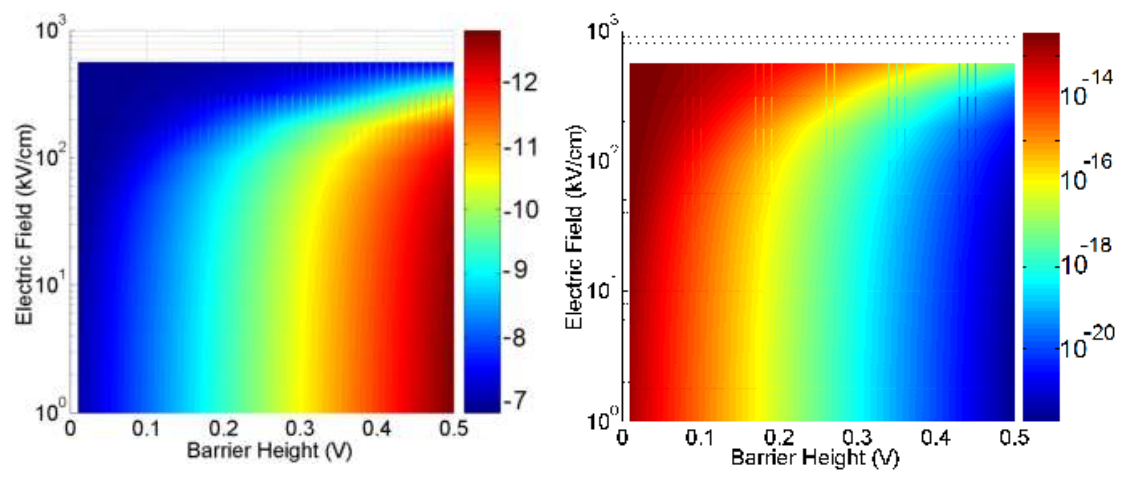

Figure 32. Left figure is a contour plot of TCR versus electric field and barrier height between CNTs of the film. The right figure shows the bolometer current also as a function of electric field and barrier height. The scales are the color bars on the right of each contour plot in units of \%TCR and amperes, respectively.

\subsubsection{CNT growth and charcterization}

In this section, we will discuss growth and characterization of carbon nanotubes with single wall (SWCNT) and multiwall (MWCNT) for use as the high performance bolometric element for development of MWIR and LWIR sensitive detector elements.
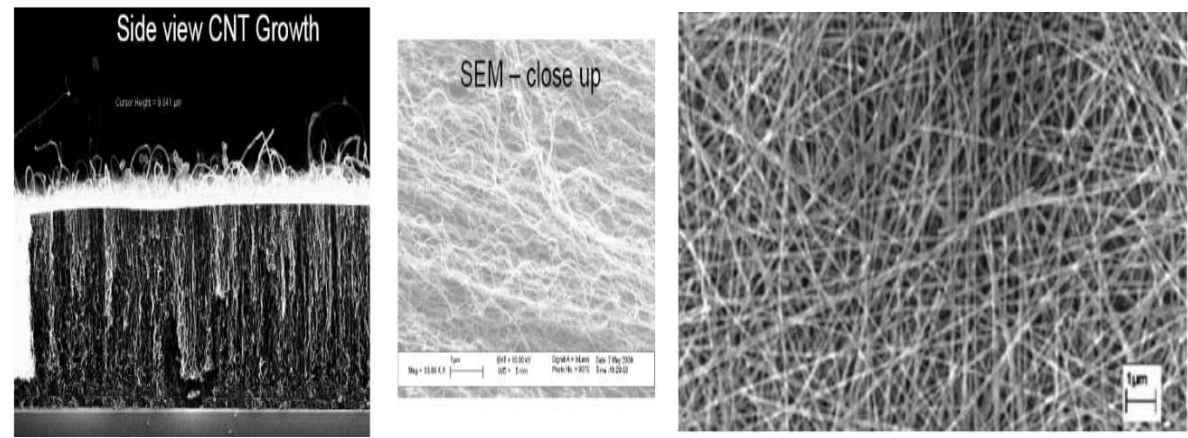

Figure 33. Growth of multiwall CNT forest with the ability to separate the form growth substrate with good length / diameter uniformity and the MWCNT released from the template.[55]

Figure 33 presents growth of dense oriented multi-walled CNT "forest like growth". The figure shows the CNT growth can be easily separated from the growth substrate. We have shown good length/diameter uniformity. Further work on the growth optimization is underway.

Figure 34 shows the prototype fixture to evaluate the CNT films for bolometric application. This fixture is being used for quick evaluation of both electrical and optical characteristics of the CNT samples. The figure also shows the preliminary results of reflectivity measurements 
for SWCNT and MWCNT samples with various sample treatments. We have carried out some preliminary measurements of TCR on CNT samples.

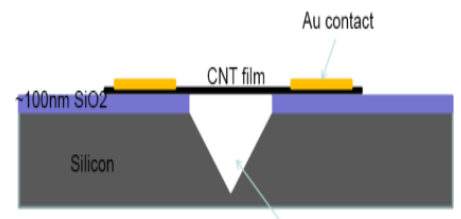

Trench made with $\mathrm{KOH}$ wet etch
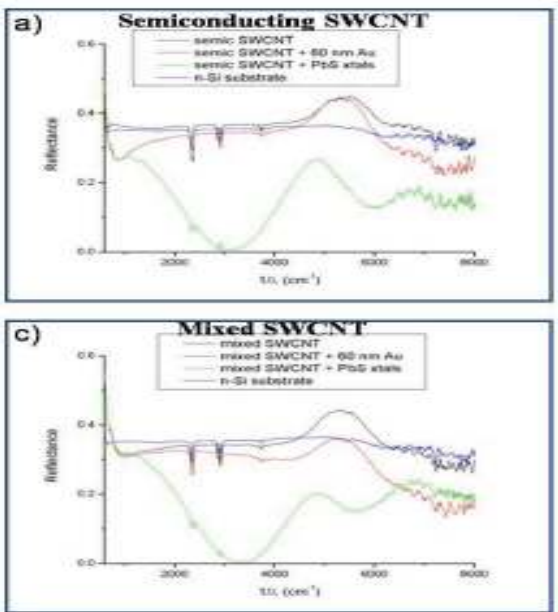
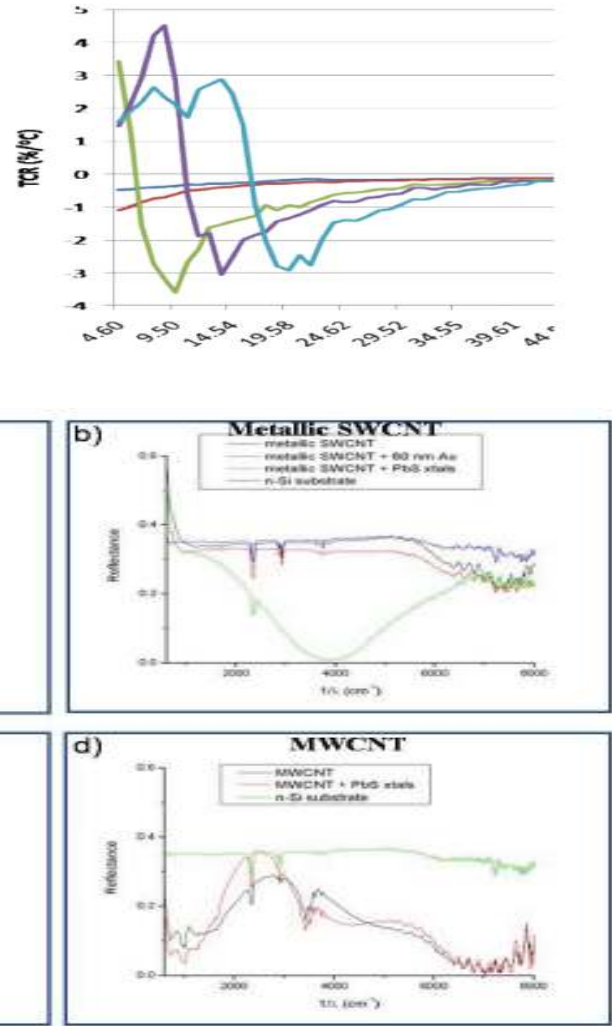

Figure 34. The composite figure shows prototype CNT film bolometer test fixture to evaluate the CNT film quality. Some preliminary data on reflectivity measurements for SWCNT and MWCNT are shown along with preliminary results on TCR measurements.[55]

Figure 35 shows scanning electron microscopy SEM. images of representative MWCNT films in the unsuspended left. and suspended.right. forms, respectively. Unlike their SWCNT counterparts, the MWCNT films contain substantial uncovered substrate areas. In addition, some minor deformation of recess is visible on suspended MWCNT films, which is similar to the SWCNT film case in the same thickness range. Figure 35.b includes a transmission electron microscopy.TEM. image of a representative individual MWCNT, which has a large hollow center of approximately $10-11 \mathrm{~nm}$ in diameter and contains approximately 40-50 CNT shells. 

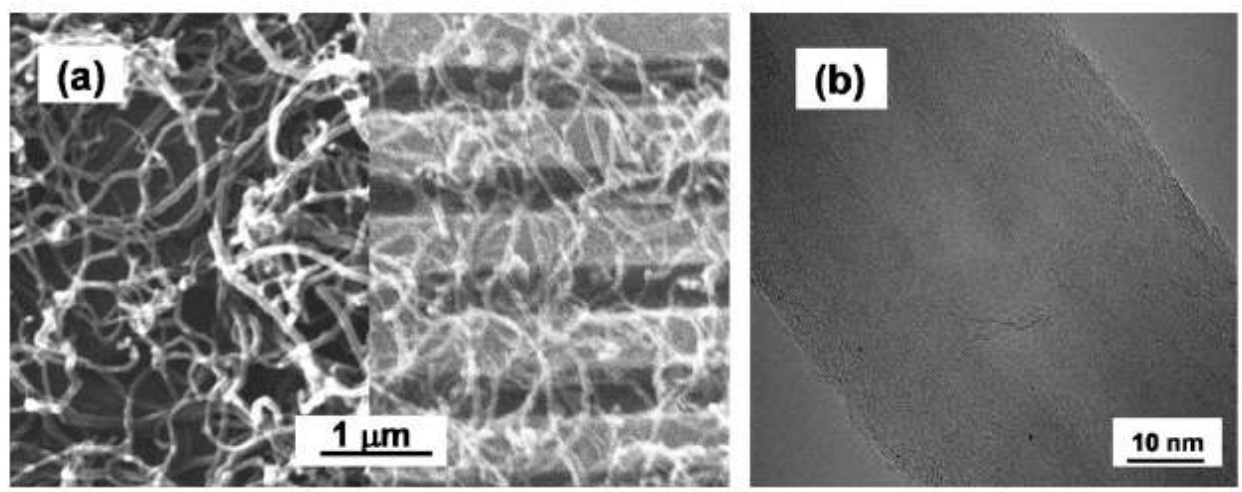

Figure 35. SEM images of unsuspended.left (a). and suspended.right (b). MWCNT films. A TEM image of a representative MWCNT. The shell number is estimated to be.40-50 for the MWCNTs [56]

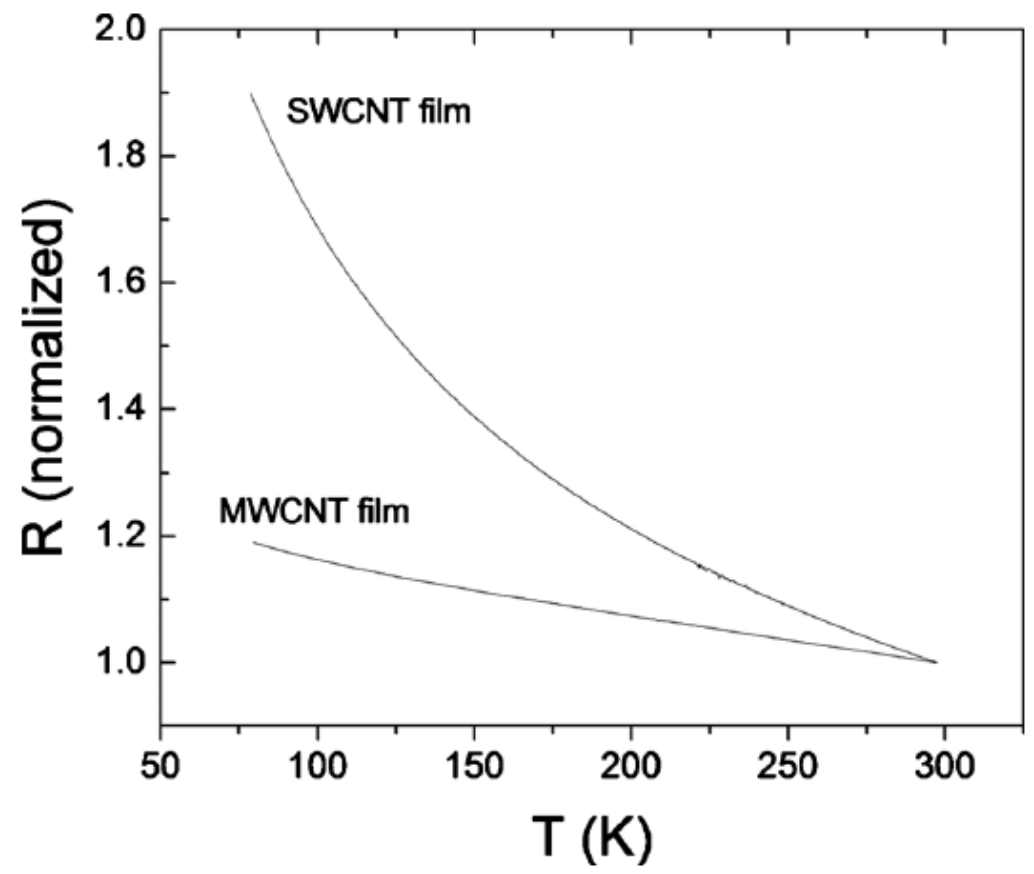

Figure 36. Resistance versus temperatures curves of SWCNT films and MWCNT films.[56]

All MWCNT films studied in this work [56] show semiconductive resistance-temperature.RT. behaviors and a representative curves is depicted in Figure 36.. Nevertheless, the increase 
in the resistivity of MWCNT films is much less than that of SWCNT films with decreasing temperature, as shown in Figure 36. This is not unexpected considering a much smaller band gap in MWCNTs. The reduced temperature dependence also implies smaller TCR absolute value in MWCNTs. For example, the TCR absolute value at room temperature for MWCNT films is about $0.07 \% / \mathrm{K}$ in contrast to. $0.17 \% / \mathrm{K}$ for SWCNT films. R-T curve after suspending the MWCNT film has been also measured.
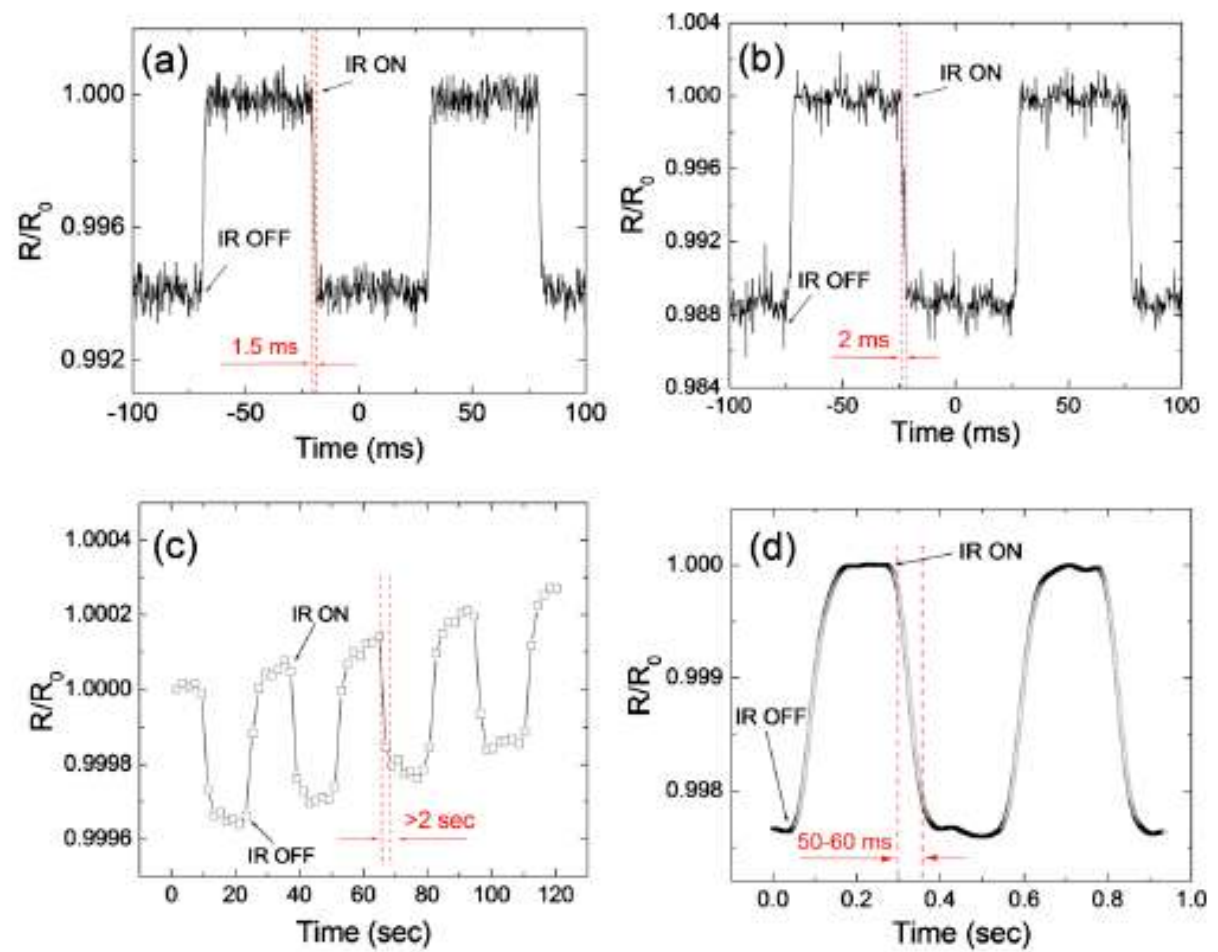

Figure 37. Photoresponse of unsuspended and suspended CNT films.(a) Unsuspended MWCNT film, $f=10 \mathrm{~Hz}$, in IR.3 $\mathrm{mW} / \mathrm{mm} 2$;.(b). suspended MWCNT film, $\mathrm{f}=10 \mathrm{~Hz}$, in IR.3 mW/mm2 ; (c) unsuspended SWCNT film, $\mathrm{f}=1 / 30 \mathrm{~Hz}$, in IR.3.5 $\mathrm{mW} / \mathrm{mm} 2$; and (d) suspended SWCNT film, $\mathrm{f}=2 \mathrm{~Hz}$, in IR. $3.5 \mathrm{~mW} / \mathrm{mm} 2$ [56].

Figure 37 compares the Photoresponse R/R0 of MWCNT films in unsuspended (a) and suspended (b) cases, where $\mathrm{R}_{0}$ is the sample resistance before IR radiation was turned on and the change in the resistance caused by IR radiation is defined as. $R=R-R_{0}$. For comparison, the results of their SWCNT counterparts are also included in Figure 37 (c).unsuspended and Fig. 37(d). suspended..

Two major differences are visible between MWCNT and SWCNT films, a significantly higher.R/ $\mathrm{R}_{0}$ and a much shorter response time in the cases of MWCNT. The.R $/ \mathrm{R}_{0}$ for MWCNT 
samples is typically in the range of a few percent, which is more than one order of magnitude higher than that of suspended SWCNT films and two orders of magnitude higher than the unsuspended SWCNT films at a comparable IR power. Considering a lower TCR absolute value in MWCNTs, the much enhanced Photoresponse of MWCNT films should be attributed to the naturally suspended inner CNT shells, which may provide an ideal configuration to enhance the bolometric effect by improving light absorption and reducing thermal link. Physical suspension of the films in both MWCNT.Fig. 37.(b) and SWCNT.Fig. 37(d.) cases results in a further improvement of.R/ $\mathrm{R}_{0}$ as compared to their unsuspended counterparts. The improvement is, however, much more pronounced in suspended cases [56].
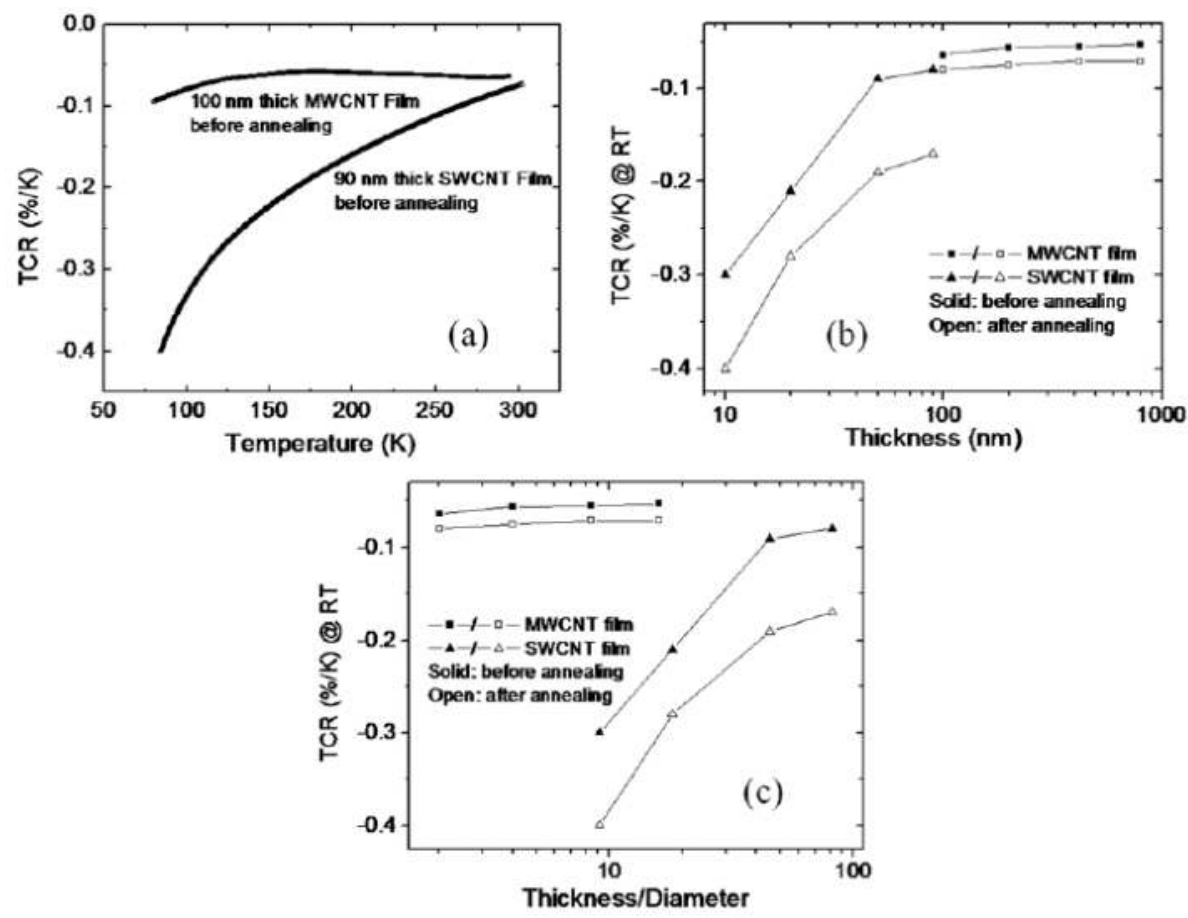

Figure 38. TCR as function of temperature for a $90 \mathrm{~nm}$ thick MWCNT film (a) and a $100 \mathrm{~nm}$ thick MWCNT film before annealing. (b) TCR versus thickness for MWCNT and SWCNT films with different thicknesses. (c) TCR versus thickness/ diameter ratios for SWCNT and MWCNT films [57].

We have also shown the results of TCR as function of temperature in figure 38, for a $90 \mathrm{~nm}$ thick MWCNT film (a) and a $100 \mathrm{~nm}$ thick MWCNT film before annealing. (b) TCR versus thickness for MWCNT and SWCNT films with different thicknesses. (c) TCR versus thickness/ diameter ratios for SWCNT and MWCNT films [57].

We have discussed recent efforts for modeling CNT based bolometer and the experimental work for development of next generation carbon nanostructure based infrared detectors and 
arrays. Our goal is to develop high performance, high frame rate, and uncooled nanobolometer for MWIR and LWIR bands. We also discussed CNT growth system and its capability to grow samples of various orientations. We have also presented recent results on SWCNT and MWCNT samples that show promise for use of CNT for developing next generation high performance small pixel bolometer arrays.

\section{Summary}

In this chapter, we have discussed recent advances in nanostructured based detector technology, materials and devices for optical sensing applications. The chapter has presented an overview of recent work underway on a variety of semiconductors and advanced materials such as $\mathrm{GaN}, \mathrm{ZnO}, \mathrm{Si} / \mathrm{SiGe}$, InGaAs and CNT for optical sensing applications.

Optical sensing technology is critical for defense and commercial applications including optical communication. Advances in optoelectronics materials in the UV, Visible and Infrared, using nanostructures, and use of novel materials such as CNT have opened doors for new approaches to apply device design methodology that are expected to offer enhanced performance and low cost optical sensors in a wide range of applications.

We have covered the UV band (200-400 nm) and address some of the recent advances in nanostructures growth and characterization using $\mathrm{GaN} / \mathrm{AlGaN}, \mathrm{ZnO} / \mathrm{MgZnO}$ based technologies and their applications. We have also discussed nanostructure based Si/SiGe technologies (400-1700 nm) that covers various bands of interest in visible-near infrared for detection and optical communication applications. The chapter has also discussed some of the theoretical and experimental results in these detector technologies.

Recent advancements in design and development of CNT based detection technologies have shown promise for optical sensor applications. We have presented theoretical and experimental results on these device and their potential applications in various bands of interest. It is anticipated that the current research and development presented in this chapter will enable a host of new integrated technologies for a variety of defense and commercial applications.

Although numerous research activities are ongoing in the area of Nanoscience and technology, we briefly made comments on such technologies to make readers aware of various research activities.

\section{Acknowledgements}

The authors gratefully acknowledge the contributions of the many distinguished scientists in the United States for development of nanotechnology based EO/IR detector technology for optical sensor applications. 


\section{Author details}

Ashok K. Sood ${ }^{1}$, Nibir K. Dhar ${ }^{2}$, Dennis L. Polla ${ }^{3}$, Madan Dubey ${ }^{4}$ and

Priyalal Wijewarnasuriya ${ }^{4}$

1 Magnolia Optical Technologies Inc., Woburn, MA, USA

2 Defense Advanced Project Agency, Arlington, VA, USA

3 College of Science and Engineering, University of Minnesota, Minneapolis, MN, USA

4 Army Research Laboratory, Adelphi, MD, USA

\section{References}

[1] Raytheon Vision Systems Spectral Charts, Goleta, CA

[2] N. K. Dhar. "IR Material Research at the Army Research Laboratory" Invited Keynote Paper, Proceedings of SPIE, Volume 6542, 65420C (2007)

[3] J.P. Long, “UV detectors and focal plane array imagers based on AlGaN p-i-n photodiodes", Opto-Electronics Review 10(4), 251-260 (2002)

[4] M. Crawford, "Advances in AlGaN-based deep UV LEDs “, MRS Proceedings, Vol. 831, fall 2004.

[5] M.B. Reine, "Solar-blind AlGaN 256x256 p-i-n detectors and focal plane arrays", Proc. of SPIE, Vol. 6119 (2006)

[6] Ashok K. Sood, Robert A. Richwine, Yash R. Puri, Nibir K. Dhar, Dennis L. Polla, and Priyalal S. Wijewarnasuriya, " Multispectral EO/IR sensor model for evaluating UV, visible, SWIR, MWIR and LWIR system performance" Proceedings of SPIE 7300, $73000 \mathrm{H}(2009)$

[7] Abdiel Rivera, John Zeller, Tariq Manzur, Ashok Sood and Mehdi Anwar, " MOCVD Growth and Characterization of ZnO Nanowire Arrays for UV Detectors" Proceedings of SPIE, Volume 8540, October 2012.

[8] Liang, S., Sheng, S., Liu, Y., Huo, Z., Lu, Y., and Shen, H., “ZnO Schottky ultraviolet photodetectors," J. Cryst. Growth 225, 110-113 (2001).

[9] Zhang, J., Que, W., Jia, Q., Ye, X., and Ding, Y., "Controllable hydrothermal synthesis of $\mathrm{ZnO}$ nanowires arrays on $\mathrm{Al}$-doped $\mathrm{ZnO}$ seed layer and patterning of $\mathrm{ZnO}$ nanowires arrays via surface modification of substrate," Appl. Surf. Sci. 257(23), 10134-10140 (2011). 
[10] Lee, C. H., Yi, G. C.., Zuev, Y. M., and Kim, P., “Thermoelectric power measurements of wide band gap semiconducting nanowires," Appl. Phys. Lett. 94, 22106 (2009).

[11] Falyouni, F., Benmamas, L., Thiandoume, C., Barjon, J., Lusson, A., Galter, P., and Sallet, V., "Metal organic chemical vapor deposition growth and luminescence of ZnO micro- and nanowires," Journal Vac. Sci. Technol. B 87, 1662 (2009).

[12] Jeong, M. C., Oh, B.Y., Lee, W., and Myoung, J. M., "Comparative study on the growth characteristics of $\mathrm{ZnO}$ nanowires and thin films by metal-organic chemical vapor deposition (MOCVD)," Journal of. Crystal Growth 268, 149-154 (2004).

[13] Kim, S. W., Fujita, S., and Fujita, S., "ZnO nanowires with high aspect ratios grown by metal-organic chemical vapor deposition using gold nanoparticles," Appl. Phys. Lett. 86,153119 (2005).

[14] Lee, W., Jeong, M. C., and Myoung, J. M., "Catalyst-free growth of ZnO nanowires by metal-organic chemical vapor deposition (MOCVD) and thermal evaporation," Acta Mat. 52, 3949-3957 (2004).

[15] Liou, S. C., Hsiao, C. S., and Chen, S. Y., "Growth behavior and microstructure evolution of $\mathrm{ZnO}$ nanorods grown on $\mathrm{Si}$ in aqueous solution," Journal of. Crystal. Growth 274, 438 (2005).

[16] Dong, J. W., Osinski, A., Hertog, B., Dabiran, A. M., Chow, P. P., Heo, Y. W., Norton, D. P, and Pearton, S. J., "Development of MgZnO-ZnO-AlGaN heterostructures for ultraviolet light emitting applications," J. Electron. Mat. 34, 416-423 (2005).

[17] Rivera, A., Zeller, J., Sood, A.K., and Anwar, A. F. M., “A Comparison of ZnO Nanowires and Nanorods Grown Using MOCVD and Hydrothermal Processes," J. Electron. Mat. 42, 894-900 (2013).

[18] Ha, B., Ham, H., and Lee, C. J., "Photoluminescence of $\mathrm{ZnO}$ nanowires dependent on $\mathrm{O}_{2}$ and Ar annealing," Phys. Chem. Solids 69, 2453-2456 (2008).

[19] Djurišić, A. B., Ng, A.M.C., and Chen, X.Y., “ZnO nanostructures for optoelectronics: Material properties and device applications," Progress Quantum Electronics 34, 191-259 (2010)

[20] Mehdi Anwar, Abdiel Rivera, Anaz Mazady, Hung Chou, John Zeller and Ashok K. Sood, "ZnO Solar Blind Detectors: from Material to System", Proceedings of SPIE Volume 8868, 8868B (2013).

[21] Zhong Lin Wang, Guang Zhu, Ya Yang, Sihong Wang and Caofeng Pan, “ Progress in Nanogenerator for Portable Electronics" Materials Today Volume 15, Number 12 December 2012

[22] Wenzhuo Wu, Xiaonan Wen and Zhong Lin Wang, “ Texel- Addressable Matrix of Vertical-Nanowire and Adaptive Tactile Imaging" Science, Volume 340, 24 May 2013. 
[23] R.D. Dupuis, H.J. Ryou and D. Yoder. "High-performance GaN and AlGaN ultraviolet avalanche photodiodes grown by MOCVD on bulk III-N substrates", Proc. of SPIE, Vol. 6739 (2006)

[24] S.C. Shen, Y. Chang, J. B. Limb, J.H. Ryou, P. D. Yoder and R.D. Dupuis, “ Performance of Deep UV GaN Avalanche Photodiodes Grown by MOCVD”, IEEE Photonics Technology Letters, Volume 19, Number 21, November 2007.

[25] Y. Zhang, S.C. Shen, H. J. Kim, S. Choi, J. H. Ryou, R.D. Dupuis and B. Narayan, "Low-Noise GaN Ultraviolet p-i-n photodiodes on GaN Substrates" Applied Physics Letters, 94, 221109 ( 2009).

[26] Ashok K. Sood, Robert A. Richwine, Roger E. Welser, Yash R. Puri, Russell D. Dupuis, Mi-Hee Ji, Jemoh Kim, Theeradetch Detchprohm, Nibir K. Dhar and Roy L. Peters, “ Development of III-N UVAPDs for Ultraviolet Sensor Applications" Proceedings of SPIE Volume 8868, 88680T (2013)

[27] Ashok K. Sood, Robert A. Richwine, Yash R. Puri, Russell D. Dupuis, Nibir K. Dhar and Raymond S. Balcerak “Development of GaN/AlGaN APD's for UV Imaging Applications" Proceedings of SPIE 7780, 77800E (2010)

[28] J.P. Long, "UV detectors and focal plane array imagers based on AlGaN p-i-n photodiodes", Opto-Electronics Review 10(4), 251-260 (2002)

[29] M. Crawford, "Advances in AlGaN-based deep UV LEDs “, MRS Proceedings, Vol. 831, fall 2004.

[30] M.B. Reine, "Solar-blind AlGaN 256x256 p-i-n detectors and focal plane arrays", Proc. of SPIE, Vol. 6119 (2006)

[31] Ashok K. Sood, Robert A. Richwine, Yash R. Puri, Nibir K. Dhar, Dennis L. Polla, and Priyalal S. Wijewarnasuriya, “ Multispectral EO/IR sensor model for evaluating UV, visible, SWIR, MWIR and LWIR system performance" Proceedings of SPIE 7300, $73000 \mathrm{H}(2009)$

[32] S.C. Shen, Y. Chang, J. B. Limb, J.H. Ryou, P. D. Yoder and R.D. Dupuis, “ Performance of Deep UV GaN Avalanche Photodiodes Grown by MOCVD”, IEEE Photonics Technology Letters, Volume 19, Number 21, November 2007.

[33] Y. Zhang, S.C. Shen, H. J. Kim, S. Choi, J. H. Ryou, R.D. Dupuis and B. Narayan, “Low-Noise GaN Ultraviolet p-i-n photodiodes on GaN Substrates" Applied Physics Letters, 94, 221109 ( 2009).

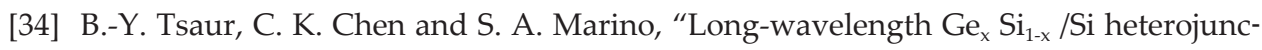
tion infrared detectors and focal plane array," Proc. SPIE 1540, 580-595, 1991.

[35] H. Wada, M. Nagashima, K. Hayashi, J. Nakanishi, M. Kimata, N. Kumada and S. Ito, "512 x512 element GeSi/Si heterojunction infrared focal plane array," Proc. SPIE 3698, 584-595, 1999. 
[36] A. K. Sood, R. A. Richwine, Y. R. Puri, N. DiLello, J. L. Hoyt, N. Dhar, R. S. Balcerak, and T. G. Bramhall, "Development of SiGe Arrays for Visible-Near IR Imaging Applications," Proc. SPIE 7780,77800F, 2010.

[37] V.T. Bublik, S.S. Gorelik, A.A. Zaitsev and A.Y. Polyakov, "Calculation on the Binding Energy of Ge-Si Solid Solution," Phys. Status Solidi 65, K79-84, 1974.

[38] A. K. Sood, R. A. Richwine, Y. R. Puri, N. DiLello, J. L. Hoyt, T. Akinwande, N. K. Dhar, R. S. Balcerak, and T. G. Bramhall, "Characterization of SiGe detector arrays for Visible-Near IR Imaging Sensor Applications," Proc. SPIE 8012,801240, 2011.

[39] Ashok K. Sood, Robert A. Richwine, Gopal Pethuraja, Yash R. Puri, Je-Ung Lee, Pradeep Haldar and Nibir K. Dhar, “ Design and Development of Wafer-Level Short Wave Infrared Micro-Camera" Proceedings of The SPIE Volume 8704, 870439 ( 2013).

[40] R.J. van der A, R.W.M. Hoogeveen, H.J. Spruijt, and A.P.H. Goede, "Low noise InGaAs infrared (1.O-2.4tm) Focal Plane Arrays," Proc. SPIE 2957, 54-65, 1997.

[41] D. Acton, M. Jack, and T. Sessler, "Large format short-wave infrared (SWIR) focal plane array (FPA) with extremely low noise and high dynamic range," Proc. SPIE 7298, 72983E, 2009.

[42] B. M. Onat, W. Huang, N. Masaun, M. Lange, M. H. Ettenberg, and C. Dries, “Ultra low dark current InGaAs technology for focal plane arrays for low-light level visibleshortwave infrared imaging," Proc. SPIE 6542, 65420L, 2007.

[43] J. Boisvert, T. Isshiki, R. Sudharsanan, P. Yuan, and P. McDonald, "Performance of very low dark current SWIR PIN arrays," Proc. SPIE 6940, 69400L, 2008.

[44] M. MacDougal, J. Geske, J. Wang, and D. Follman, "Short-wavelength infrared imaging using low dark current InGaAs detector arrays and vertical-cavity surface-emitting laser illuminators," Optical Engineering 50(6), 061011, 2011.

[45] A. D. Hood. "Large InGaAs Focal Plane Arrays for SWIR Imaging" Proceedings of SPIE, Volume 8353, 83530A, 2012.

[46] H. Yuan, M. Meixell, J. Zhang, P. Bey, J. Kimchi, and L.C. Kilmer "Low Dark Current Small Pixel Large Format InGaAs 2-D Photodetector array Development" Proceedings of SPIE, Volume 8353, 835309, 2012.

[47] H. Yuan, G. Apgar, J. Kim, J. Laquindanum, V. Nalavade, P. Beer, J. Kimchi, and T. Wong, "FPA development: from InGaAs, InSb, to HgCdTe," Proc. SPIE 6940, 69403C, 2008 .

[48] R. Blackwell, D. Lacroix, T. Bach, et. al “ 17 micron microbolometer FPA Technology at BAE Systems" Proceedings of SPIE, Volume 7298,72980P ( 2009).

[49] C. Li, G. Skidmore, C. Howard, E. Clarke and J. Han, “Advancement in 17-micron pixel pitch uncooled focal plane arrays" Proceedings of SPIE, Volume 7298, 72980S (2009). 
[50] T. Schimert, C. Hanson, J. Brady, et. al. " Advanced in small-pixel, large-format alpha-silicon bolometer arrays", Proceedings of SPIE, Volume 7298, 72980 T (2009)

[51] C. Trouilleau, B. Fieque, S. Noblet, F. Giner et.al. "High Performance uncooled-amorphous silicon TEC less XGA IRFPA with 17 micron pixel pitch" Proceedings of SPIE Volume 7298, 72980Q, (2009).

[52] E. Pop, D. Mann, Q. Wang, K. Goodson, H. Dai, Thermal Conductance of an Individual Single-Wall Carbon Nanotube above Room Temperature Nano Letters 6, 96 (2006)

[53] A. Akturk, N. Goldsman, G. Metze, "Self-consistent modeling of heating and MOSFET performance in 3-D integrated circuits," IEEE Trans. on Elect. Dev. 52 (11): 2395-2403 (2005).

[54] Ashok K. Sood, E. James Egerton, Yash R. Puri, Gustavo Fernandes, Jimmy Xu, Akin Akturk, Neil Goldsman, Nibir K. Dhar, Madan Dubey, Priyalal S. Wijewarnasuriya and Bobby I Lineberry, " Design and Development of CNY based Micro-bolometer for IR Imaging Applications" Proceedings of SPIE, Volume 8353, 83533A, May 2012

[55] Gustavo Fernandes, Jin Ho Kim, Jimmy Xu, Ashok K. Sood, Nibir K. Dhar and Madan Dubey, "Unleashing Giant TCR from Phase -Changes in Carbon Nanotube Composites" Proceedings of SPIE Volume 8868, 88680S, September 2013

[56] Rongtao Lu, Jack J. Shi, F. Javier Baca and Judy Z. Wu, “ High Performance Multiwall Carbon Nanotube Bolometer" Journal of Applied Physics, 108, 084305 ( 2010).

[57] Rongtao Lu, Rayyan Kamal and Judy Z. Wu, “ A comparative study of $1 / \mathrm{f}$ noise and temperature coefficient of resistance in multiwall and single-wall carbon nanotube bolometers" nanotechnology, Volume 22, 265503 (2011). 